\title{
Micropeptide ASAP encoded by LINC00467 promotes colorectal cancer progression by directly modulating ATP synthase activity
}

\author{
Qiwei Ce, , ${ }^{1,2,3}$ Dingjiacheng Jia, ${ }^{1,2}$ Dong Cen, ${ }^{4}$ Yadong Qi, ${ }^{2,5}$ Chengyu Shi, ${ }^{3,6}$ Junhong Li, ${ }^{3,6}$ Lingjie Sang, ${ }^{3,6}$ Luo-jia Yang, ${ }^{3,6}$ Jiamin He, ${ }^{2,5}$ \\ Aifu Lin, ${ }^{3,6,7}$ Shujie Chen, ${ }^{2,5,7}$ and Liangjing Wang ${ }^{1,2,7}$ \\ 'Department of Gastroenterology, Second Affiliated Hospital of Zhejiang University School of Medicine, Hangzhou, Zhejiang Province, China. ${ }^{2}$ Institution of Gastroenterology, Zhejiang University, Hangzhou, \\ Zhejiang Province, China. ${ }^{3}$ MOE Laboratory of Biosystem Homeostasis and Protection, College of Life Sciences, Zhejiang University, Hangzhou, Zhejiang Province, China. ${ }^{4}$ Department of Ceneral Surgery and \\ ${ }^{5}$ Department of Gastroenterology, Sir Run Run Shaw Hospital, Zhejiang University, Hangzhou, Zhejiang Province, China. ${ }^{6}$ Key Laboratory for Cell and Gene Engineering of Zhejiang Province, Zhejiang, China. \\ ${ }^{7}$ Cancer Center, Zhejiang University, Hangzhou, Zhejiang Province, China.
}

\begin{abstract}
Emerging evidence has shown that open reading frames inside long noncoding RNAs (IncRNAs) could encode micropeptides. However, their roles in cellular energy metabolism and tumor progression remain largely unknown. Here, we identified a 94 amino acid-length micropeptide encoded by IncRNA LINC00467 in colorectal cancer. We also characterized its conservation across higher mammals, localization to mitochondria, and the concerted local functions. This peptide enhanced the ATP synthase construction by interacting with the subunits $\alpha$ and $\gamma$ (ATP5A and ATP5C), increased ATP synthase activity and mitochondrial oxygen consumption rate, and thereby promoted colorectal cancer cell proliferation. Hence, this micropeptide was termed ATP synthase-associated peptide (ASAP). Furthermore, loss of ASAP suppressed patient-derived xenograft growth with attenuated ATP synthase activity and mitochondrial ATP production. Clinically, high expression of ASAP and LINC00467 predicted poor prognosis of colorectal cancer patients. Taken together, our findings revealed a colorectal cancerassociated micropeptide as a vital player in mitochondrial metabolism and provided a therapeutic target for colorectal cancer.
\end{abstract}

\section{Introduction}

Colorectal cancer (CRC) is the second leading cause of cancerrelated mortality globally (1). The prognosis of patients with early CRC has been greatly improved in the past decade (2). However, the survival of patients with advanced CRC remains poor. A comprehensive understanding of molecular events in CRC progression and discovery of therapeutic targets may improve the prognosis of CRC patients.

Long noncoding RNAs (lncRNAs), once considered as "transcriptional noise," have been demonstrated as broadly functioning via diverse regulatory mechanisms $(3,4)$. Emerging evidence has uncovered the translational noise, that is, in addition to the classic start codons of mRNA that can translate peptides on the ribosome, the open reading frames (ORFs) inside lncRNAs could also encode micropeptides (5-7). It has been reported that micropeptides play functional roles in calcium pump regulation (8), mTOR signaling transduction (9), $\mathrm{m}^{6} \mathrm{~A}$ recognition (10), and tumor progression (11). Notably, translational profiling indicated that a considerable number of micropeptides are mitochondria located or closely related to mitochondrial functions (5). Moreover, recent studies

Authorship note: QG, DJ, and DC contributed equally to this work.

Conflict of interest: The authors have declared that no conflict of interest exists.

Copyright: @ 2021, American Society for Clinical Investigation.

Submitted: July 6, 2021; Accepted: September 23, 2021; Published: November 15, 2021

Reference information: J Clin Invest. 2021;131(22):e152911.

https://doi.org/10.1172/JCI152911 have revealed that LINCO0116 encodes a highly conserved single-pass transmembrane peptide that is located in the mitochondria and regulates fatty acid $\beta$ oxidation (12) and mitochondrial respiration $(13,14)$, highlighting micropeptides as crucial players in cellular metabolism regulation. However, little is known about the function of IncRNA-derived micropeptides in human CRC. Characterization of the micropeptides will provide insights into the molecular regulatory network in the progression of CRC.

Cancer cells undergo alterations in metabolic pathways to support their unrestricted growth, which has been recognized as one of the hallmarks of cancer (15). Recent studies have revealed that, in addition to aerobic glycolysis, many cancer cells can also employ the mitochondrial oxidative phosphorylation (OXPHOS) pathway for ATP production $(16,17)$. Notably, CRC might not be a type of tumor that solely relies on the Warburg effect, since the OXPHOS is upregulated in CRC cells (18). An in situ study of CRC further proved that total glycolytic capacity of CRC cells and the peripheral normal colon cells were similar, but the respiratory rate of CRC cells was much higher (19). The increased mitochondrial DNA (mtDNA) could promote the progression of microsatellite-stable CRC by enhancing OXPHOS (20). The OXPHOS electron transport chain (ETC) consists of 4 complexes (complexes I through IV) and transfers electrons from donors derived from the TCA cycle and fatty acid oxidation (21). ATP synthase, also known as ETC complex V, converts ADP to ATP under the proton gradient (22). Some compounds targeting complex V have been screened for preclinical research $(23,24)$. Insight into the mechanism of the 
OXPHOS pathway may shed light on the discovery of therapeutic targets for CRC treatment.

In this study, we reported that ATP synthase-associated peptide (ASAP), a LINC00467-encoded 94 aa micropeptide, was involved in mitochondrial metabolism and ATP production in CRC progression. ASAP bound to ATP synthase subunits $\alpha$ and $\gamma$ (ATP5A and ATP5C) and enhanced their interaction, thereby further promoting ATP synthase activity and cell proliferation. Clinically, high ASAP expression predicted poor prognosis of CRC patients.

\section{Results}

Characterization of the coding potential of CRC-associated lncRNAs. Emerging evidence shows functional peptides could be encoded by short ORFs within lncRNAs (7). To identify lncRNAs with coding potential in CRC, we analyzed a ribosome-sequencing data set (Gene Expression Omnibus [GEO] GSE143263) of HCT116 CRC cells; 318 lncRNAs with ribosome-interacting potential were identified (Figure 1A and Supplemental Table 1; supplemental material available online with this article; https://doi.org/10.1172/ JCI152911DS1). We then compared those candidates with a group of RNAs that were highly expressed in CRC from a data set (GEO GSE22598) and included in a previously reported lncRNA-derived micropeptides database (ref. 5, Figure 1A, and Supplemental Table 1). This analysis showed 6 differentially expressed lncRNA candidates in CRC cells, which was verified in The Cancer Genome Atlas (TCGA) database (Figure 1B). Among those 3 highly upregulated lncRNAs, LINCO0467 was the most enriched in the polysome of HCT116 cells (Figure 1C).

LINCO0467 was originally annotated as a long intergenic noncoding RNA (NR_026761.2). We analyzed the expression of LINC00467 in the GEO databases (GSE22598, GSE37364) and found that the expression of LINC00467 was much higher in CRC tissues as compared with adjacent normal tissues (Figure 1D and Supplemental Figure 1A). Nuclear-cytoplasm fractionation assays indicated that LINCO0467 was a cytoplasmic RNA in both HCT116 and RKO cells (Supplemental Figure 1, B and C), further supporting its potential to encode peptides.

LINC00467 encodes an uncharacterized peptide: ASAP. There were 5 ORFs more than 25 aa within the LINCO0467 transcript according to ORF-finder analysis (ref. 25 and Supplemental Table 2). To determine the translational potential of those ORFs, we generated C-terminal Flag-tagged constructs for ORF1-5 and transfected them into HCT116 cells. Western blot analysis showed that only the ORF1 (196-480 nt) of LINC00467 could code a micropeptide (Figure 1E), which was consistent with previous reports that the first ORF at the $5^{\prime}$ region of the sense strand is most likely to be translated $(26,27)$.

To determine whether the start codon of ORF1 was functional, we generated an ORF1-Flag construct by fusing Flag-tag at the C-terminus of ORF1 (Figure 1F). Those Flag fusion proteins were detected with immunofluorescence staining (Figure 1G) and Western blot analysis (Supplemental Figure 1D), but were not expressed in start codon-perturbed ORF1 mutants (ATG to ATT; Figure $1 G$ and Supplemental Figure 1D). Furthermore, we established a series of constructs by fusing a Mut-GFP (the start codon ATGGTG mutated to ATTGTT) to the C-terminus of ORF1 (Supplemental Figure 1E). GFP fusion proteins could be observed with a microscope and detected by Western blot analysis in WtORF1Mut-GFP-transfected CRC cells (Supplemental Figure 1, F and G). However, mutation of the ORF1 start codon (ATG to ATT) abolished the expression of GFP fusion proteins (Supplemental Figure $1, F$ and $\mathrm{G})$. Taken together, these results indicated the start codon of LINC00467 ORF1 was functionally recognized.

ORF1 of LINC00467 encoded a 94 aa micropeptide, which was highly enriched with basic residues (Supplemental Figure 2A). The secondary structure of the peptide was predicted by $3 \mathrm{D}$ modeling (C-I-TASSER). Results demonstrated that there were $\alpha$-helixes at the C-terminus (Supplemental Figure 2B). Sequence comparisons showed that the aa sequence was highly conserved among higher mammals (Supplemental Figure 2C), and there were no matching proteins and known domains/motifs in the 94 aa peptide, indicating an uncharacterized peptide. We termed it ATP synthase-associated peptide (ASAP) according to the function and mechanism studied.

The ASAP peptide is endogenously expressed. To confirm that ASAP is endogenously expressed, we inserted a Flag-tag at the C-terminal of the ORF1 (Flag-KI) using the CRISPR/Cas9 system (ref. 28 and Figure 1H). The Flag signal could be detected by immunofluorescence staining (Figure 1I) and Western blot analysis (Supplemental Figure $1 \mathrm{H}$ ). We further generated an antibody against the ASAP peptide and determined the specificity of the ASAP antibody. The expression levels of ASAP can be depleted by siRNAs targeting the LINC00467 transcript in HCT116 cells (Supplemental Figure 3, A and B). In addition, the ASAP levels in different CRC cell lines were detected by immunofluorescence staining (Figure 1J and Supplemental Figure 3C) and Western blot (Supplemental Figure $3 \mathrm{D})$. We further transfected a $3 \times$ Flag-ASAP construct $(3 \times$ Flag peptide was fused to the C-terminus of ASAP) into HCT116 and RKO cells and detected endogenous ASAP and exogenous FlagASAP in the same immune blot (Supplemental Figure 3E). Collectively, our data showed that ASAP was endogenously expressed.

ASAP is an inner mitochondrial membrane-associated micropeptide. The subcellular localization of protein is closely related to its function $(29,30)$. To gain insight into the function of ASAP, we performed Gene Ontology (GO) enrichment analysis on genes that are coexpressed with LINC00467 in the TCGA COAD database. Interestingly, the result showed that LINC00467 was positively correlated with genes related to mitochondrial protein complexes (Figure 2A). Considering the distribution pattern of ASAP in immunofluorescence staining (Figure 1J and Supplemental Figure 3C), we hypothesized that ASAP was localized in the mitochondria. Though bioinformatic analysis (MITOPROT) indicated that ASAP lacks a mitochondrial-targeting sequence (MTS), ASAP had a high density of positively charged residues that could promote mitochondrial import (ref. 31 and Supplemental Figure 2A). As expected, ASAP was observed to be colocalized with MitoTracker, a mitochondria-specific fluorescent dye (Figure 2B and Supplemental Figure 4, A-C).

The mitochondria were purified as we previously reported (32). The purity of mitochondria was assessed using antibodies specific to mitochondrial (SDHA and TOM20) and cytoplasmic (vinculin and $\alpha$-tubulin) proteins, and ASAP was enriched in the mitochondria fraction (Figure 2C and Supplemental Figure 4D). To further assess the mitochondrial localization of ASAP, the mitochondria 


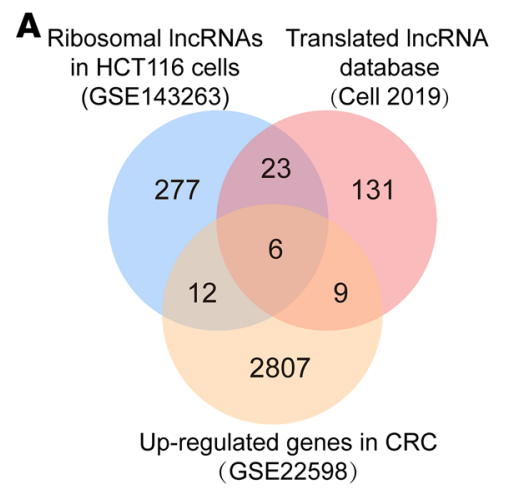

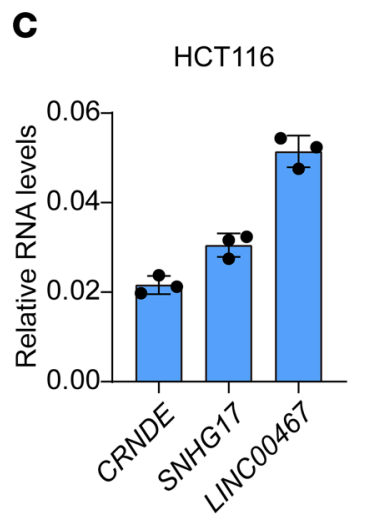

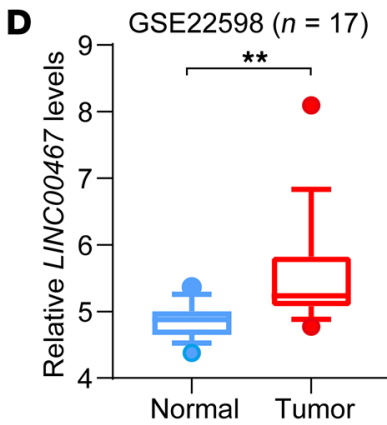

E

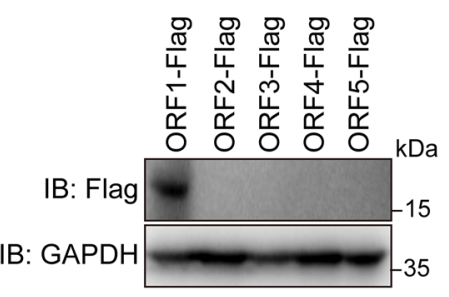

B

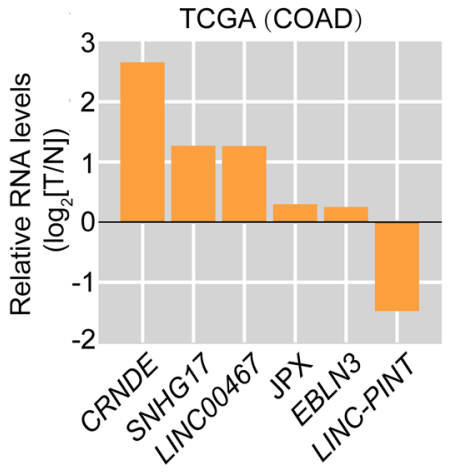

G

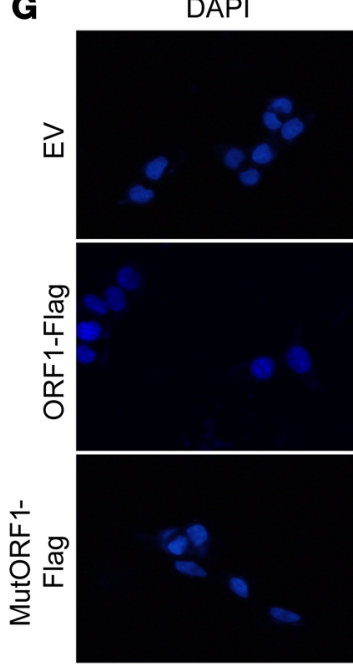

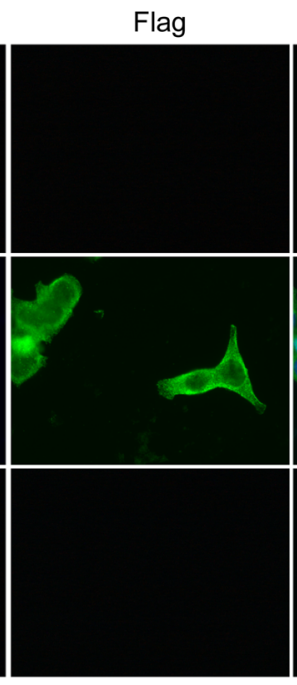

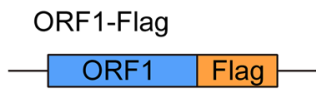

MutORF1-Flag

ATG $\rightarrow$ ATT

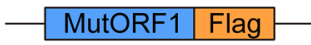

\section{H}

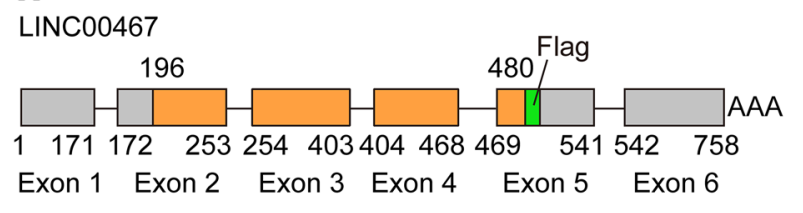

I

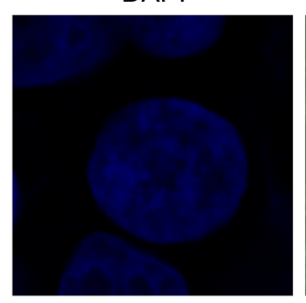

Flag

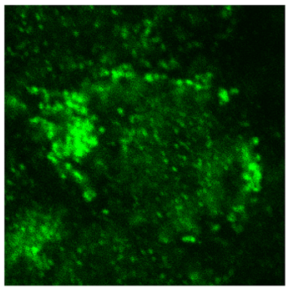

Merge

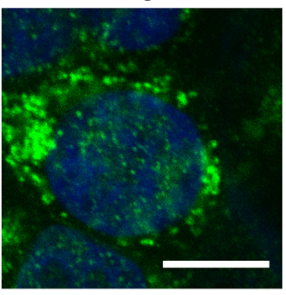

J

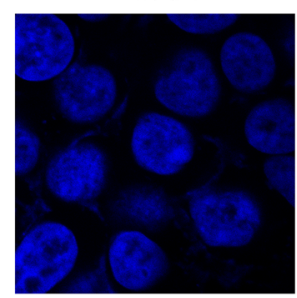

ASAP

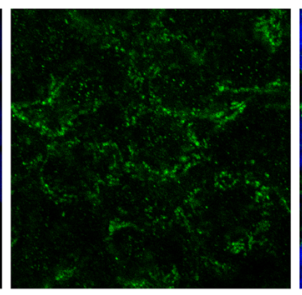

Merge

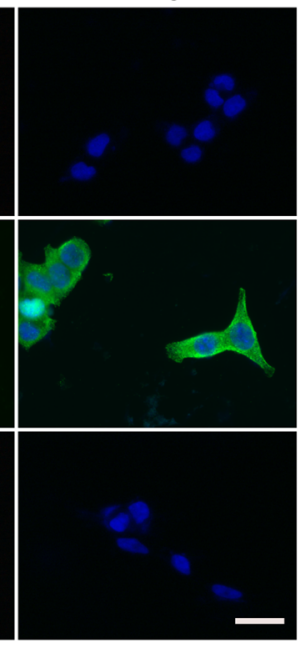

Merge

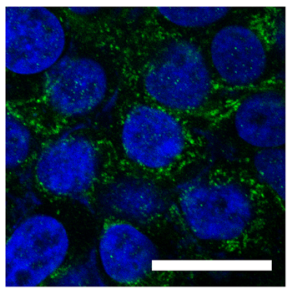

Figure 1. LINC00467 encodes an uncharacterized peptide, ASAP. (A) A Venn diagram showing upregulated genes in CRC (CSE22598), ribosomal IncRNAs in HCT116 cells (CSE143263), and translated IncRNA from a previously reported database. (B) The expression of 6 candidate IncRNAs in the TCGA COAD database. (C) Relative levels of 3 candidate IncRNAs in HCT116 cells were determined by polysome profiling followed by RT-qPCR. Data are presented as mean \pm SD. $n=3$ biologically independent experiments. (D) Data set from GSE22598 indicates LINC00467 expression levels in CRC tissues and adjacent normal tissues $(n=17)$. Data are presented as a box plot with box and whiskers. Bounds of box show the 25th and 75 th percentiles, and the central lines in the box represent the median value. Whiskers show 10th to 90th percentiles. Outlying values, 5.37, 4.38 (N), 8.09, 4.77 (T). Paired samples, 2-sided Student's $t$ test. ${ }^{* *} P<0.01$. (E) Five ORF-Flag fusion constructs were transfected into HCT116 cells, and ORF-Flag fusion protein was detected by Western blot analysis. (F) Diagram of Flag fusion constructs. Start codon of ORF1 was mutated to ATT. (C) Indicated constructs were transfected in HCT116 cells, and Flag signal (green) was detected by immunofluorescence. Nuclei were stained with DAPI (blue). Scale bar: $10 \mu \mathrm{m}$. (H) Diagram of ORF1 location at the LINC00467 locus and the Flag-tag that was inserted to the end of ORF1. The 6 exons and Poly(A) tail of LINC00467 are shown. (I) ORF-Flag fusion protein (green) was detected by immunofluorescence in HCT116 cells. Nuclei were stained with DAPI (blue). Scale bar: $10 \mu \mathrm{m}$. (J) Expression of ASAP peptide (green) in HCT116 cells was detected by immunofluorescence with prepared anti-ASAP antibody. Nuclei were stained with DAPI (blue). Scale bar: $10 \mu \mathrm{m}$. Data are representative of 3 independent experiments (E, G, I, and J).

were purified and subjected to proteolysis with serial dilutions of proteinase $\mathrm{K}(\mathrm{PK})$. The outer mitochondrial membrane (OMM) protein TOM20 was sensitive to proteolysis at lower PK concentrations (12) than the inner mitochondrial membrane-associated (IMM- associated) protein ATP5A. We found that ASAP exhibited a degradation pattern similar to that of ATP5A (Figure 2D and Supplemental Figure 4E). The mitochondria were also exposed to PK proteolysis in the presence or absence of the detergent Triton, and the results 
A

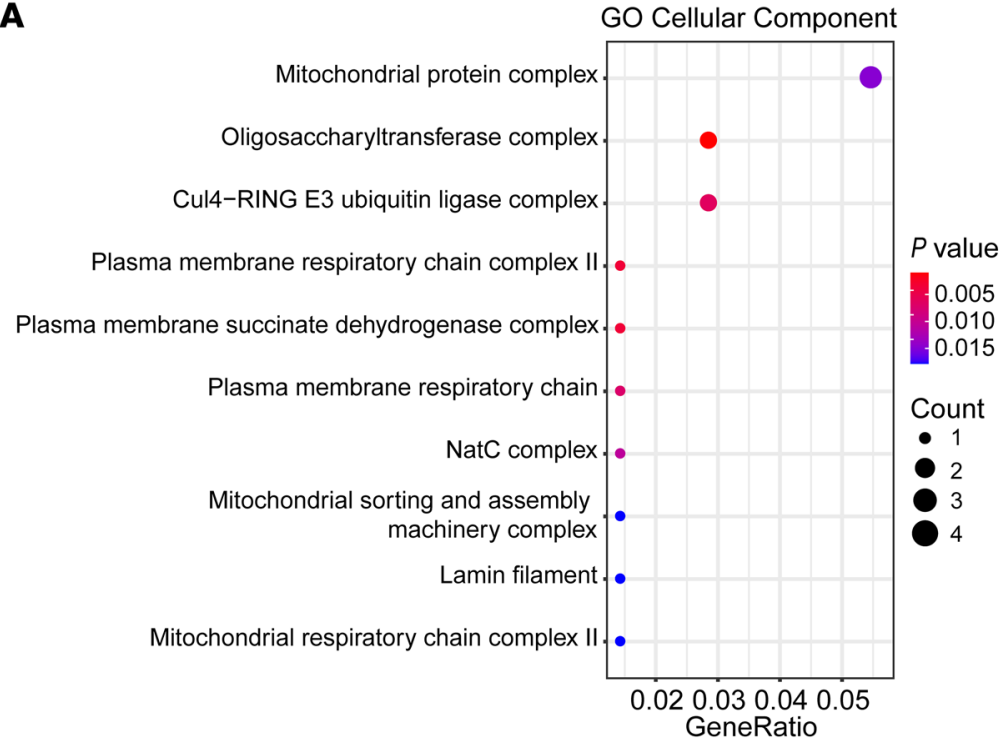

B

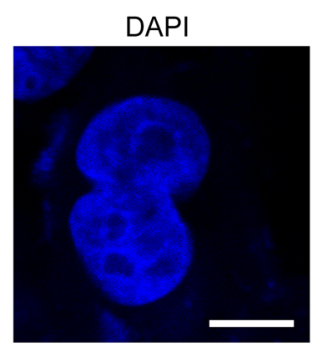

MitoTracker

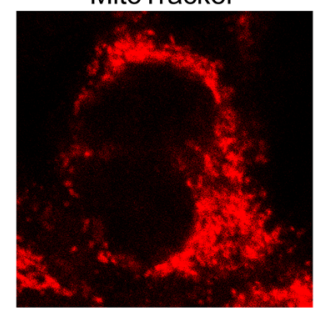

HCT116

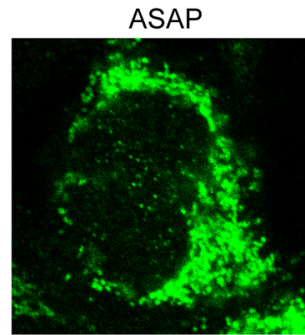

Merge

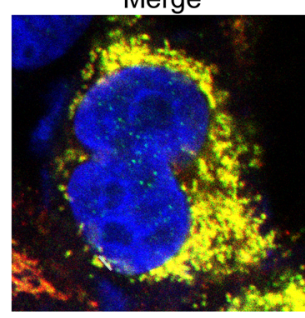

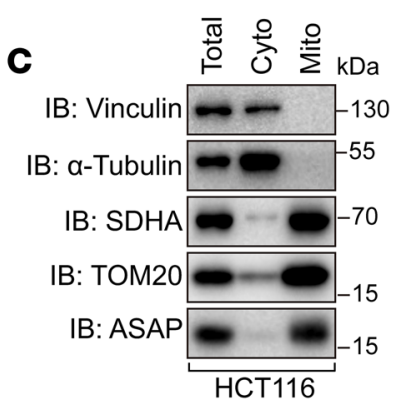

$\mathbf{F}$

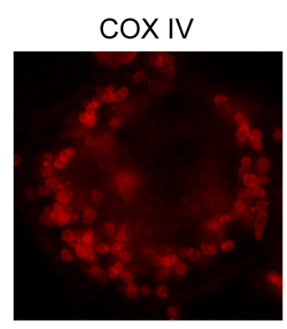

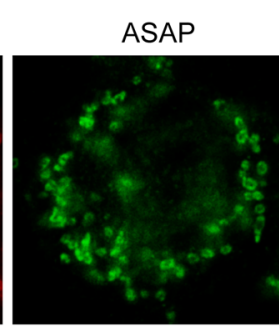

D

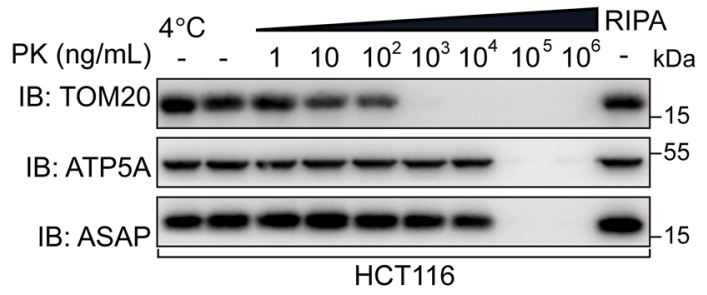

G

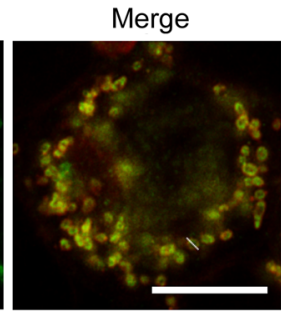

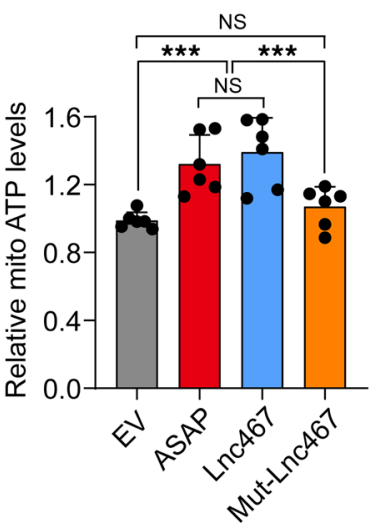

E

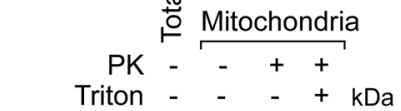

IB: TOM20

IB: ATP5A

IB: ASAP

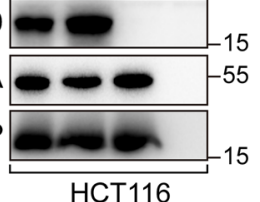

H

HCT116

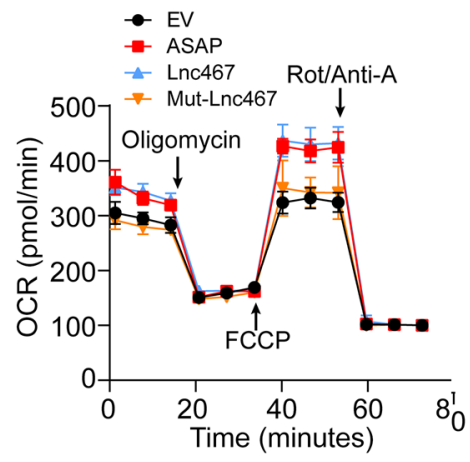

Figure 2. ASAP is localized to mitochondria and related to mitochondrial ATP production. (A) GO analyses of genes coexpressed with LINC00467 in TCGA COAD database. (B) Immunofluorescence of ASAP (green) and MitoTracker (red) in HCT116 cells. Nuclei were stained with DAPI (blue). Scale bar: $10 \mu \mathrm{m}$. (C) Western blot detection of ASAP in the purified mitochondria from HCT116 cells with the indicated protein markers (SDHA and TOM20 for mitochondria and vinculin and $\alpha$-tubulin for the cytosol). Cyto, cytosol; Mito, mitochondria. (D) Mitochondria were isolated from HCT116 cells and subjected to PK digestion at the indicated concentrations at $37^{\circ} \mathrm{C}$. Mitochondria lysed in RIPA buffer served as a control for the total mitochondrial fraction. Western blots were performed for markers of OMM (TOM20), IMM (ATP5A), and ASAP. (E) Mitochondria were isolated from HCT116 cells and subjected to PK (10³ $\mathrm{ng} / \mathrm{mL}$ ) proteolysis in the presence or absence of detergent (1\% Triton). Western blots were performed for markers of OMM (TOM20), IMM (ATP5A), and ASAP. (F) Immunofluorescence staining was performed to mark ASAP (green) and IMM marker COX IV (red). The section was visualized using Leica TCS SP8 microscope with Lightning mode. Scale bar: $10 \mu \mathrm{m}$. (G) Relative mitochondrial ATP production was detected. Indicated HCT116 cells were treated with recording buffer (with $5 \mathrm{mM}$ 2-DG and $5 \mathrm{mM}$ pyruvate) to determine ATP generation under mitochondrial ATP synthesis. Data are presented as mean values \pm SD from $n=6$ biologically independent experiments. One-way ANOVA followed by Tukey's test. ${ }^{* *} P<0.001$. (H) OCR profile was monitored in indicated HCT116 cells with a Seahorse XF24 analyzer. The metabolic inhibitors were injected at different time points, as indicated. Data are presented as mean values \pm SD. $n=3$ biologically independent experiments. Data are representative of 3 independent experiments (B-F). 
indicated that ASAP was resistant to PK treatment and solubilized by the Triton (refs. 33, 34, Figure 2E, and Supplemental Figure 4F). Immunofluorescence staining showed that ASAP was colocalized with IMM marker COX IV and distributed along the periphery of the IMM, with limited residual in the mitoplast (Figure $2 \mathrm{~F}$ and Supplemental Figure 4G). Considering that ASAP colocalized to IMM and presented little potential as a membrane-integrated protein (analyzed by TMHMM server, data not shown), ASAP should be a mitoplast protein and peripherally attached to the IMMs.

ASAP regulates mitochondrial ATP production. To determine the functional role of ASAP in CRC, we generated a series of cell lines. ASAP, LINC00467, or Mut-LINC00467 (in which the start codon of ASAP mutated to ATT) was overexpressed in both HCT116 and RKO cells, respectively. In addition, 2 ASAP-KO cell lines were generated using a CRISPR/Cas9-mediated homologous recombination technique (ref. 28 and Supplemental Figure $3 \mathrm{~F})$. We found that genomic mutation at the start codon of ASAP abolished the translation and protein levels of ASAP, but the transcription of LINC00467 was not affected (Supplemental Figure 3, G-I). ASAP-KO cells were further reconstituted with LINC00467 or Mut-LINC00467, respectively (Supplemental Figure 3, J-M).

The 5 complexes required for the mitochondrial OXPHOS process are all located in the IMM; among them, complexes I-IV form the ETC. Complex IV consumes oxygen, and complex V converts ADP to ATP (35). Given that ASAP peripherally attached to the IMM, we assessed the effect of ASAP on mitochondrial respiratory and ATP production. We found that overexpression of ASAP could increase mitochondrial ATP production as well as ATP levels in the whole cell (Figure $2 \mathrm{G}$ and Supplemental Figure 5, A, D and E), while these effects were abolished in ASAP-KO cells (Supplemental Figure 5, B, C, F, and G). Oxygen consumption rate (OCR) in CRC cells was evaluated by Seahorse mitochondria stress assay. We found that overexpression of ASAP increased OCR before administration with oligomycin, the ATP synthase inhibitor, suggesting the function of ASAP in regulating basal respiration (Figure $2 \mathrm{H}$ and Supplemental Figure $5 \mathrm{H}$ ). Meanwhile, spare respiratory capacity (the difference between basal respiration and the maximum respiratory capacity after FCCP treatment) was increased in ASAP-overexpressed cells (Figure $2 \mathrm{H}$ and Supplemental Figure $5 \mathrm{H}$ ). ASAP KO significantly reduced the basal respiration and spare respiratory capacity of CRC cells (Supplemental Figure 5, I and J). In conclusion, our results indicate that ASAP might be involved in mitochondrial ATP production.

$A S A P$ promotes $C R C$ proliferation in vitro and in vivo. We then carried out CCK-8 assay and colony formation assay to evaluate cell proliferation. Both ASAP and LINC00467 were able to promote cell proliferation, while these effects were attenuated in mutated LINC00467-transfected cells (Figure 3, A and B, and Supplemental Figure 6, A and B). Similarly, ASAP KO in HCT116 and RKO cells suppressed cell proliferation, and the capability of cell proliferation could be restored by overexpression of ASAP or LINC00467 transcript, while Mut-LINC00467 failed to rescue this phenotype (Figure 3, C and D, and Supplemental Figure 6, C and D). These results suggest that LINC00467 promoted CRC cell proliferation mainly through ASAP.

We next examined the role of ASAP in CRC development in vivo. Both ASAP and LINC00467 overexpression were able to sig- nificantly promote xenograft CRC tumor growth (Figure 3, E-H, and Supplemental Figure 7, A and B) with increased cell proliferation (indicated by Ki-67 staining, Supplemental Figure 7, C-E). In contrast, ASAP KO in both HCT116 and RKO cells resulted in impaired xenograft tumor growth (Figure 3, I-L, and Supplemental Figure 7, F and G).

Additionally, we found that ASAP was downregulated in a normal intestinal epithelial cell line (NCM460; ref. 36) as compared with CRC cells (Supplemental Figure 8A). ASAP was also located to mitochondria in NCM460 cells (Supplemental Figure 8, B and C). Overexpression of ASAP was able to promote cell proliferation and ATP production in NCM460 cells (Supplemental Figure 8, D and E). However, knocking down of ASAP could not induce remarkable proliferation and ATP production changes (Supplemental Figure 8, F and G), which was different from the suppressing effect in tumor cells.

ASAP interacts with ATP5A and ATP5C and regulates ATP synthase activity. In order to identify potential regulators of mitochondria function in ASAP-interactome, we performed an immunoprecipitation assay followed by mass spectrometry (MS) analysis (Figure 4A). IMM-associated proteins were remarkably enriched in the eluate from ASAP-overexpressed HCT116 cells (Supplemental Table 3). Among these proteins, ATP5A, ATP5B, and ATP5C are the components of ATP synthase (22); SLC25A3 functions as a transport of phosphate groups and copper (37); and HADHA is the $\alpha$ subunit of mitochondrial trifunctional enzyme, which is involved in the mitochondrial $\beta$-oxidation pathway (38). We then tested the ASAP-binding potential of those candidate proteins individually and found that only ATP5A and ATP5C bound to ASAP (Figure 4, B and C, and Supplemental Figure 9, A-C). The endogenous binding of ASAP to ATP5A and ATP5C was confirmed in different cell lines (Figure 4D and Supplemental Figure 9D). ATP synthase, also known as ETC complex V, is responsible for the generation of ATP through phosphorylation of ADP under the proton gradient across the IMM (22). ATP synthase is a large protein complex composed of 2 parts, Fo and F1, of which the F1 domain assumes the catalytic function and is composed of 5 subunits, $\alpha, \beta, \gamma, \delta$, and $\varepsilon$ (39). We further tested the binding of ASAP to the 5 subunits of the F1 domain and found that ATP5A and ATP5C rather than other subunits could bind to ASAP (Supplemental Figure 9E). We purified recombinant ASAP, ATP5A, and ATP5C to perform a mutual in vitro pulldown assay, which confirmed the direct interaction between ASAP and ATP5A or ATP5C (Figure 4, $\mathrm{E}$ and F). Given the fact that ATP5A and ATP5C are in the same complex, we further tested the simultaneous presence of ASAP, ATP5A, and ATP5C within that complex. Sequential immunoprecipitation assays confirmed that ASAP formed a complex with ATP5A and ATP5C (Figure 4G).

We next asked how ASAP affected the function of ATP synthase. As ASAP did not alter the expression of ATP5A and ATP5C (Supplemental Figure 9F), we hypothesized that ASAP bridges the interaction between ATP5A and ATP5C. Co-IP assays revealed that overexpressed ASAP could enhance the interaction between ATP5A and ATP5C (Figure 5A), while loss of ASAP impaired their interactions (Figure $5 B$ ). In vitro pulldown assay confirmed that ASAP could directly promote the interaction between ATP5A and ATP5C (Figure 5C). Functionally, our results demonstrated that 
A
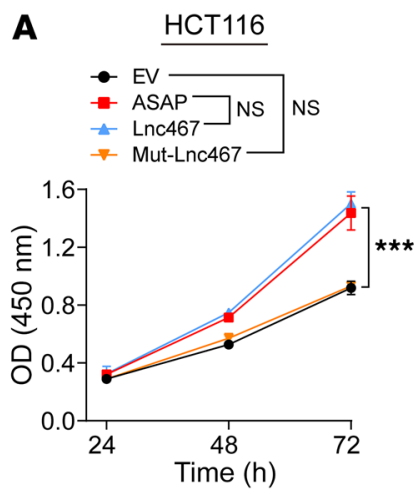

E

HCT116

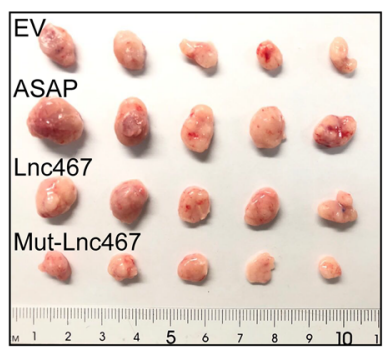

I

HCT116

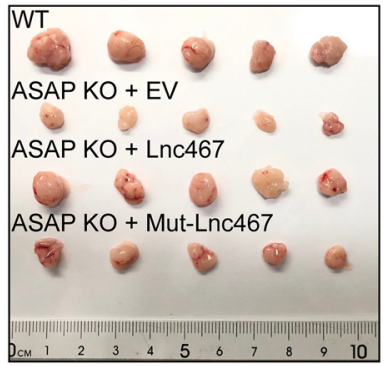

B
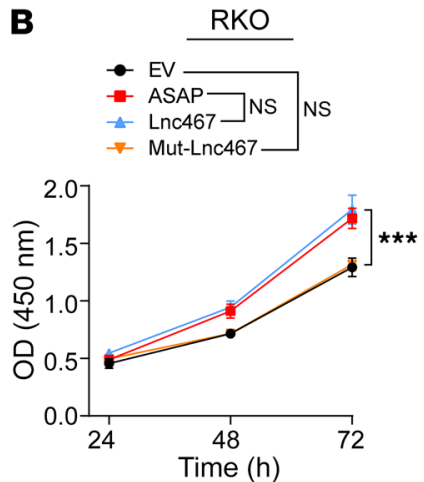

$\mathbf{F}$

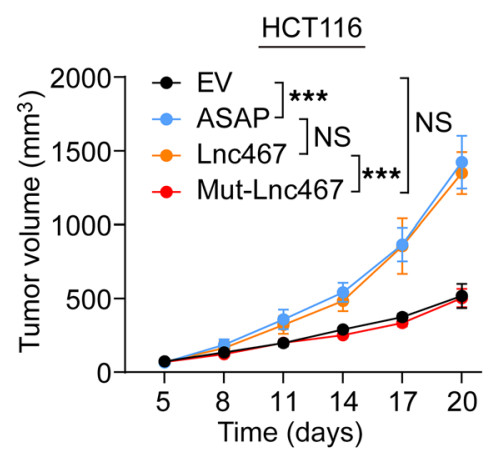

J

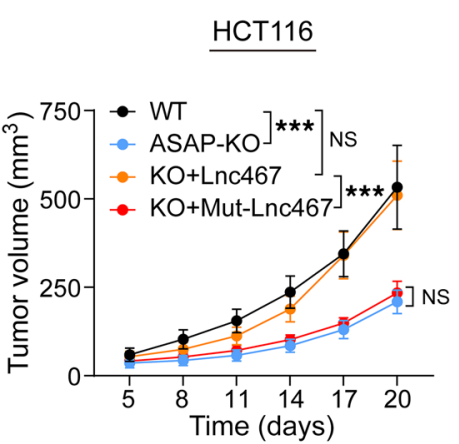

C
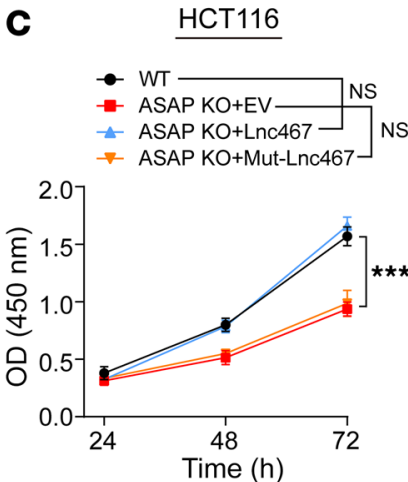

G

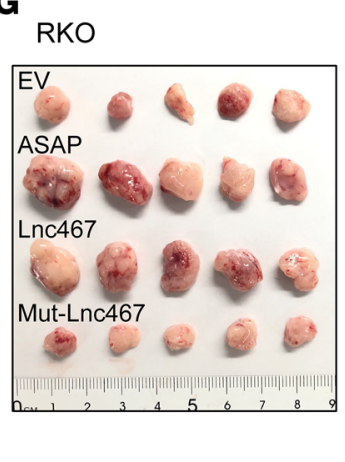

$\mathbf{K}$

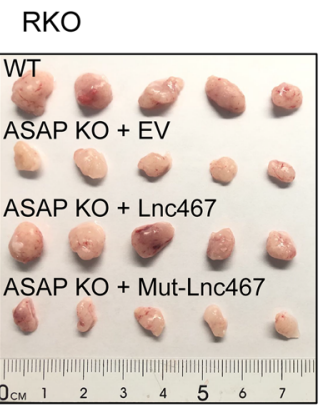

H

$\mathbf{L}$
D
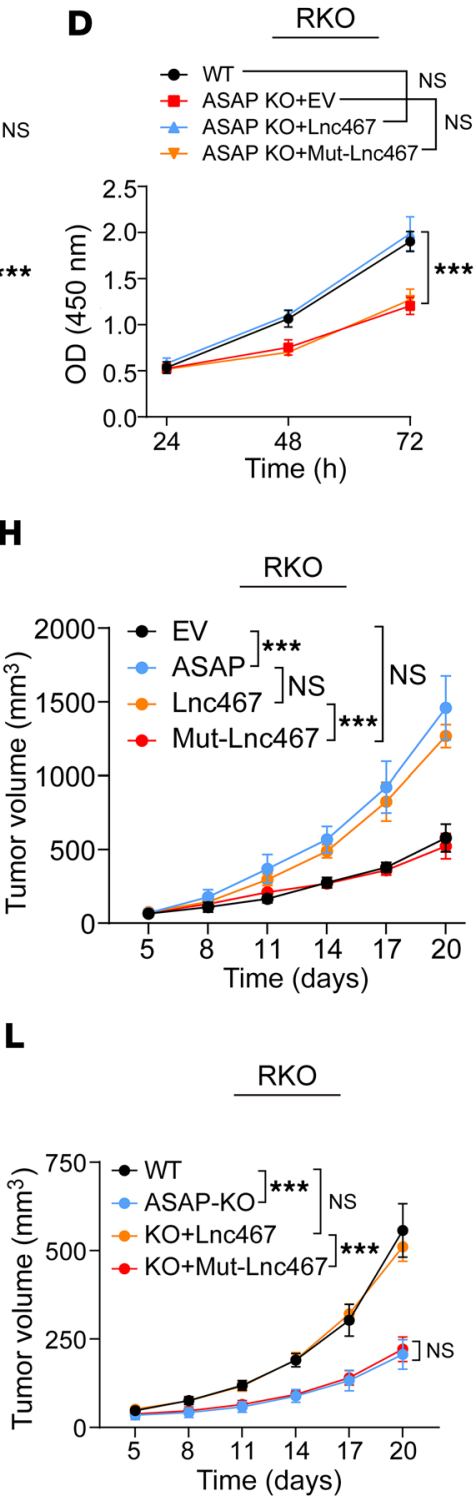

Figure 3. ASAP promotes CRC cell proliferation in vitro and in vivo. (A-D) The CCK-8 assay was used to determine the proliferation rate of indicated cells. Data are presented as mean values $\pm \mathrm{SD} . n=3$ biologically independent experiments. Two-way ANOVA analysis. ${ }^{* *} P<0.001$. (E and $\left.\mathbf{F}\right)$ Xenograft mouse model using EV control or ASAP, LINC00467-overexpressing (Lnc467), or Mut-LINC00467-overexpressing (Mut-Lnc467) HCT116 cells. In vivo generated tumors $(\mathbf{E})$ and analyses of tumor growth and volume $(\mathbf{F})$ are shown. Data are presented as mean \pm SD from $n=5$ mice per group. Two-way ANOVA. ${ }^{* * *} P<$ 0.001. (G and $\mathbf{H})$ Xenograft mouse model using EV control or ASAP, LINC00467-overexpressing (Lnc467), or Mut-LINC00467-overexpressing (Mut-Lnc467) RKO cells. In vivo generated tumors (C) and analyses of tumor growth and volume $(\mathbf{H})$ are shown. Data are presented as mean $\pm S D$ from $n=5$ mice per group. Two-way ANOVA analysis. ${ }^{* *} P<0.001$. (I and J) Xenograft mouse model using WT, ASAP-KO, LINC00467-restored (Lnc467), or Mut-LINC00467restored (Mut-Lnc467) HCT116 cells. In vivo generated tumors (I) and analyses of tumor growth and volume (J) are shown. Data are presented as mean \pm SD from $n=5$ mice per group. Two-way ANOVA analysis. ${ }^{* *} P<0.001$. (K and $\left.\mathbf{L}\right)$ Xenograft mouse model using WT, ASAP-KO, LINC00467-restored (Lnc467), or Mut-LINC00467-restored (Mut-Lnc467) RKO cells. In vivo generated tumors (K) and analyses of tumor growth and volume (L) are shown. Data are presented as mean \pm SD from $n=5$ mice per group. Two-way ANOVA analysis. ${ }^{* *} P<0.001$.

ASAP could enhance ATP synthase activity (Figure 5, D and E, and Supplemental Figure 9, G and H), which was consistent with increased mitochondrial ATP production and OCR in ASAP-overexpressed cells. Collectively, ASAP played a vital role in the regulation of ATP synthase activity through enhancing ATP5A and ATP5C interaction (Figure 5F).

Identification of key residues that contribute to ASAP-ATP synthase interaction. To further demonstrate the interaction between ASAP and ATP synthase, we conducted molecular docking simulation
(40) using the predicted ASAP structure (Supplemental Figure 2B) and Bos taurus ATP synthase structure (41) (4ASU in the PDB database), which is highly homologous with Homo sapiens ATP synthase (Supplemental Figure 10A). K78 and K79 residues of ASAP were shown to interact with D411 residue of ATP5A (D454 in H. sapiens ATP5A) by establishing salt bridges and hydrogen bond interactions (Supplemental Figure 10B). In addition, D65 residue of ASAP was shown to be involved in the binding to K172 residue in ATP5C (K197 in H. sapiens ATP5C) by hydrogen bond interactions (Supplemental 


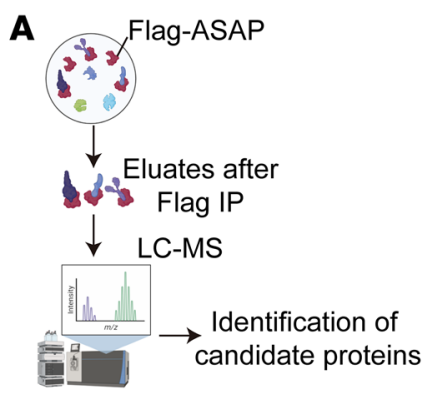

E

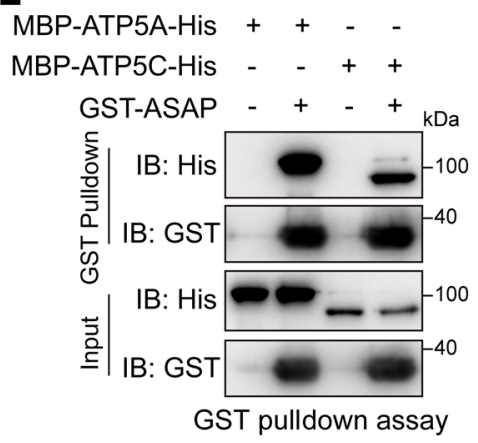

B

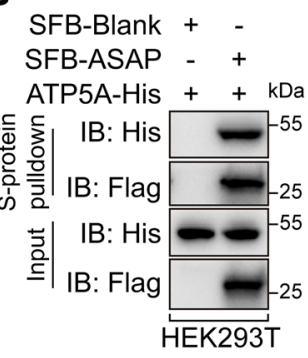

C

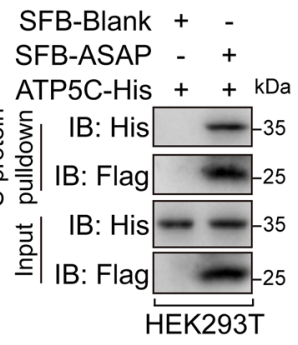

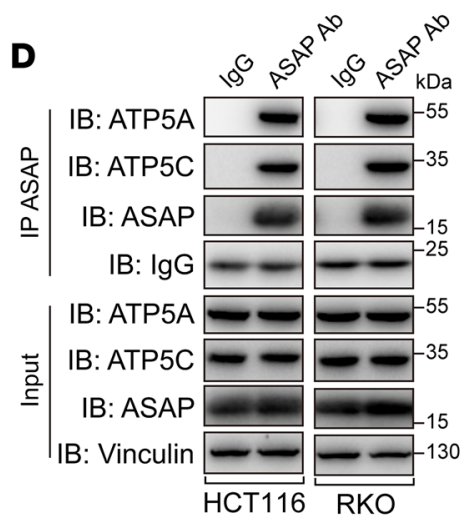

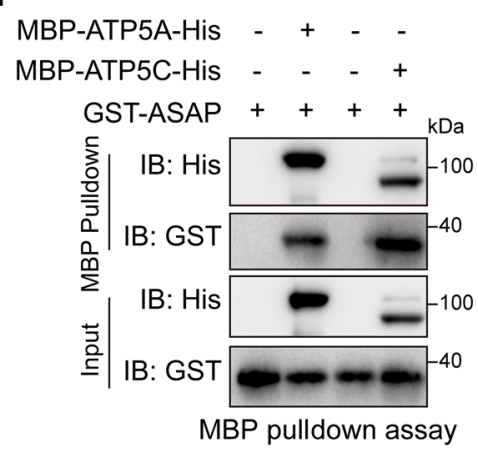

G

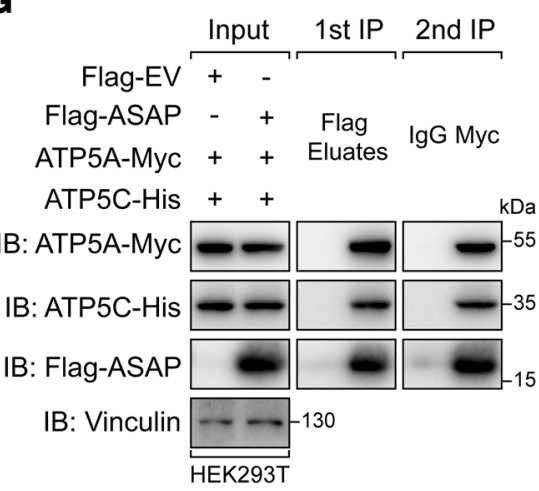

Figure 4. ASAP interacts with ATP5A and ATP5C. (A) Schematic diagram showing the identification of ASAP-interacting proteins using MS analysis following immunoprecipitation. (B and C) SFB-ASAP and ATP5A-His (B) and ATP5C-His (C) were cooverexpressed in HEK293T cells. Co-IP assays were performed to detect the interaction in ASAP-ATP5A (B) and ASAP-ATP5C (C). (D) Co-IP assays showing the endogenous interaction between ASAP and both ATP5A and ATP5C in HCT116 and RKO cells. (E and F) In vitro pulldown assays were performed using bacterial purified MBP-ATP5A-His, MBP-ATP5C-His, and CST-ASAP to confirm the direct interaction between ASAP and both ATP5A and ATP5C. (G) HEK293T cells transfected with the indicated constructs were used for sequential immunoprecipitation assay with Flag-tag immunomagnetic beads, followed by anti-Myc antibody. Data are representative of 3 independent experiments (B-G).

Figure 10C). We found that K79 residue of ASAP rather than K78 was involved in the interaction between ASAP and ATP5A (Figure $6 \mathrm{~A}$ ), and the D65 residue of ASAP was confirmed to contribute to the interaction between ASAP and ATP5C (Figure 6B).

The electrical mutations at the corresponding residues of ATP5A and ATP5C also proved the key roles of the abovementioned hydrogen bonds and salt bridges in the interaction between ASAP and ATP5A or ATP5C (Figure 6, C and D). As shown in Supplemental Figure 2C, K79 and D65 in ASAP are conserved in multiple species. In addition, ASAP-D65A did not affect its interaction with ATP5A, and ASAP-K79A did not affect its interaction with ATP5C (Supplemental Figure 10, D and E), indicating that ATP5A and ATP5C interacted with ASAP via different residues. We observed that ASAP with dual mutations (Mut-ASAP, D65A, and K79A) did not interact with either ATP5A or ATP5C, but preserved its mitochondrial localization (Supplemental Figure $10, \mathrm{~F}-\mathrm{I})$. ASAP with dual mutations failed to increase the interaction between ATP5A and ATP5C (Figure 6, E-G).

$A S A P$ regulates $C R C$ growth mainly through mitochondrial ATP production. We determined whether ASAP could promote tumor growth through binding to ATP synthase. Mut-ASAP was impotent in promoting ATP synthase activity (Figure 7, A and B) and mitochondrial and whole cell ATP production (Figure 7, C and D, and Supplemental Figure 11, A and B) as well as cell proliferation (Figure 7, E and F). WT ASAP but not Mut-ASAP could restore the phenotype in ASAP-KO HCT116 and RKO cells (Supplemental Figure 11, C-J). Meanwhile, Mut-ASAP (K79A and D65A), unable to bind to ATP5A and ATP5C, failed to promote xenograft tumor growth (Figure 7, G and H, and Supplemental Figure 11K) and ATP synthase activity (Supplemental Figure 11L) in vivo.

ATP synthase inhibitors have shown antitumor effects in preclinical studies $(23,24)$. We intervened in xenograft tumor growth using the classical ATP synthase inhibitor oligomycin. Oligomycin treatment could significantly inhibit the growth of CRC, indicating mitochondrial ATP production was essential for CRC growth in vivo (Figure 7, I and J, and Supplemental Figure 12, A-D). When the activity of ATP synthase was inhibited, the tumor-promoting effect of ASAP was significantly blocked (Figure 7, I and J, and Supplemental Figure 12, A-D). Given that adding ATP to the culture medium could replenish depleted ATP levels $(42,43)$, we tested the effect of ATP on ASAP-KO cell lines. The inhibition of cell growth in ASAP-KO cell lines could be largely rescued by supplying additional ATP (Figure 7, $\mathrm{K}$ and L, and Supplemental Figure 12, E and F). Collectively, the above results suggest that ASAP promoted tumor proliferation mainly by its regulation of mitochondrial ATP production. 

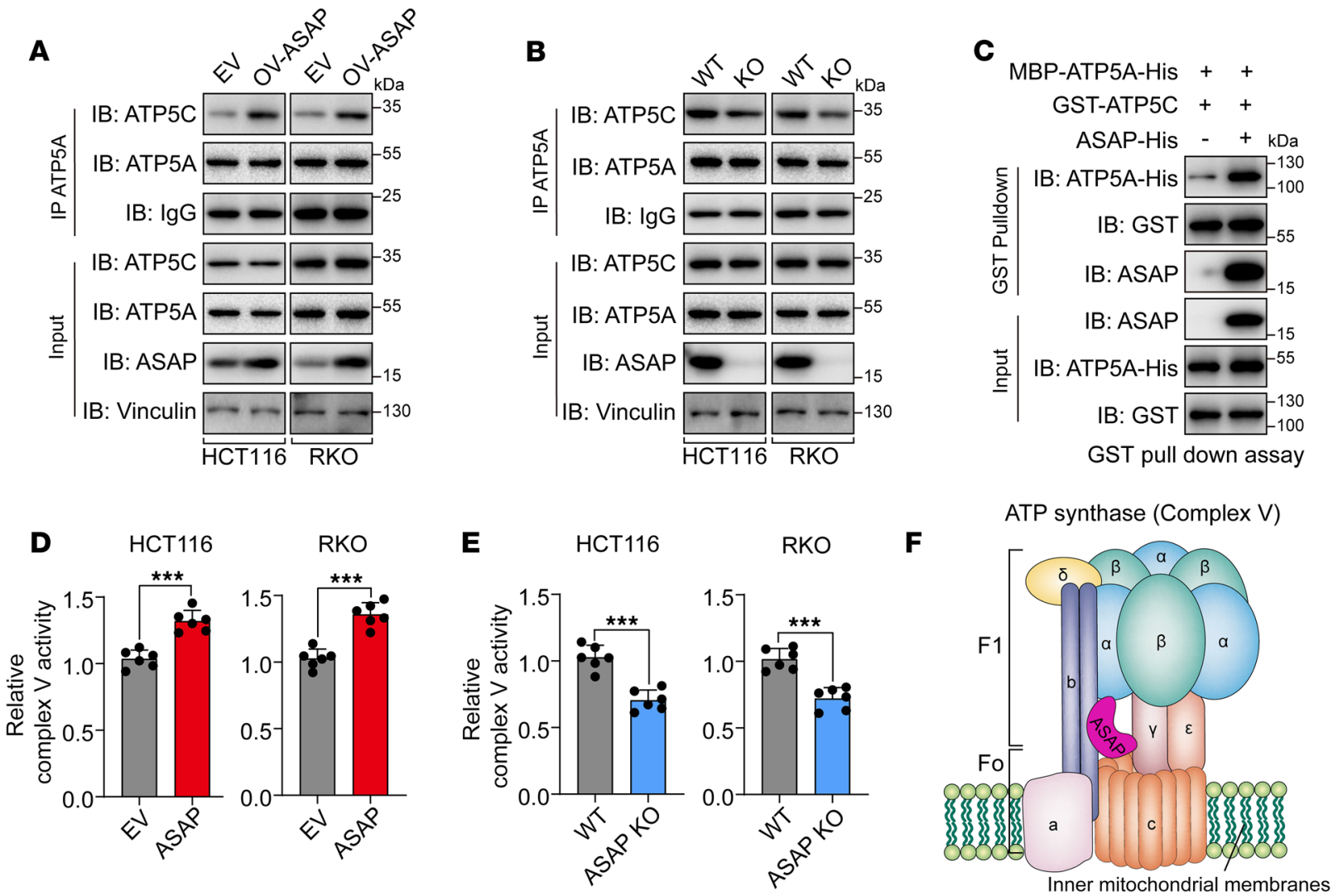

Figure 5. ASAP regulates ATP synthase activity and enhances the interaction between ATP5A and ATP5C. (A and B) The interaction between ATP5A and ATP5C was detected using Co-IP assays in HCT116 and RKO cells with overexpressed ASAP (A) or ASAP KO (B). (C) In vitro pulldown assay was performed using bacterial purified MBP-ATP5A-His and GST-ATP5C in the presence or absence of ASAP-His to confirm that ASAP directly enhances the interaction between ATP5A and ATP5C. ( $D$ and E) The activities of respiratory complex V (ATP synthase) were investigated in HCT116 and RKO cells with ASAP overexpressed (D) or ASAP KO (E). Data are presented as mean value \pm SD. $n=6$ biologically independent experiments. Independent samples; 2 -sided Student's $t$ test. ${ }^{* *} P<0.001$. (F) Schematic diagram showing the interaction between ASAP and ATP synthase. The ATP synthase structural model was constructed based on Yoshida et al. (67). Data are representative of 3 independent experiments (A-C).

Targeting ASAP suppresses the growth of CRC patient-derived xenografts. The CRISPR/Cas9 system has shown therapeutic potential (44). We designed a CRISPR/Cas9-based strategy to KO ASAP in patient-derived xenografts (PDX), as previously reported (45). Three days after subcutaneous tumor inoculation, mice received 3 weeks of intratumoral injection of either ASAP-targeted CRISPR/Cas9 (Cas9-sgASAP) vector plus delivery buffer or CRISPR v2 vector plus delivery buffer as control (Figure 8A). The tumor size in mice injected with Cas9-sgASAP vector was significantly reduced as compared with that of mice injected with control vector (Figure 8, B and C, and Supplemental Figure 13, A and B). The KO efficiency of ASAP was confirmed by Western blot analysis (Supplemental Figure 13, C and D). The Cas9-sgASAP injection group showed decreased proliferative ability, as indicated by the staining of Ki-67 and cyclin D1 (Figure 8, D and E). Loss of ASAP impaired angiogenesis, as indicated by the staining of CD31 (an endothelial cell marker) (Figure 8, D and E). Meanwhile, loss of ASAP attenuated the ATP synthase activity in PDX mice (Supplemental Figure 13, E and F), which was in line with the results of the in vitro study (Figure $5 \mathrm{E}$ ).

The expression of ASAP and LINCO0467 predicts poor outcomes in CRC patients. To uncover the clinical relevance of LINC00467 and ASAP, we first assessed LINC00467 RNA levels in 50 primary $C R C$ tissues and their matched adjacent noncancerous tissues (cohort 1). The expression of LINC00467, ATP5A, and ATP5C mRNA was significantly upregulated in CRC tissues (Figure 9, $\mathrm{A}-\mathrm{C}$ ). We then divided the participants into 2 groups (LINCO0467low and LINC00467-high) based on the RNA level of LINCO0467 compared with the median value of that of all individuals and found that the expression of LINCOO467 was positively related to RNA levels of ATP5A and ATP5C (Figure 9, D and E).

The protein levels of ASAP were higher in tumor than in matched adjacent noncancerous tissues by Western blot analysis (cohort 1; Figure 9F). Furthermore, we performed immunohistochemistry staining on a tissue microarray with 92 CRC patients (cohort 2). ASAP is widely expressed in epithelial tissues and is mainly expressed in the cytoplasm, and quantitative analysis indicated that ASAP was highly expressed in CRC tissues as compared with the noncancerous tissues (Figure 9, G and H). Consistently, the protein levels of ATP5A and ATP5C were upregulated in CRC tissues (Supplemental Figure 14, A and B). Tumors with higher ASAP expression showed relative high levels of Ki-67, ATP5A, and ATP5C (Figure 9, I-L). We also detected the expression pattern of ASAP in normal colonic tissues. ASAP was mainly expressed in the 
A

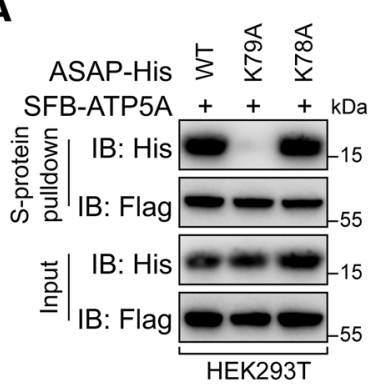

B

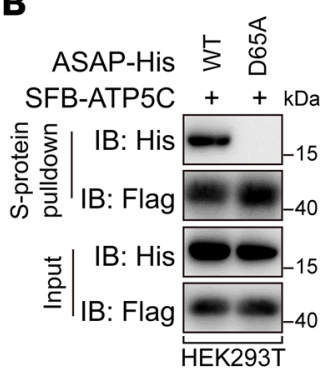

C

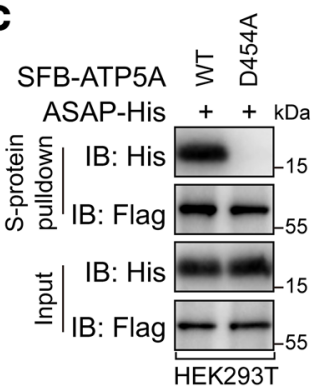

D

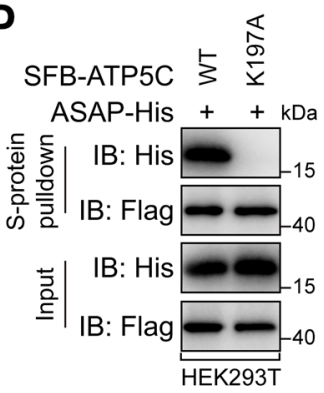

E

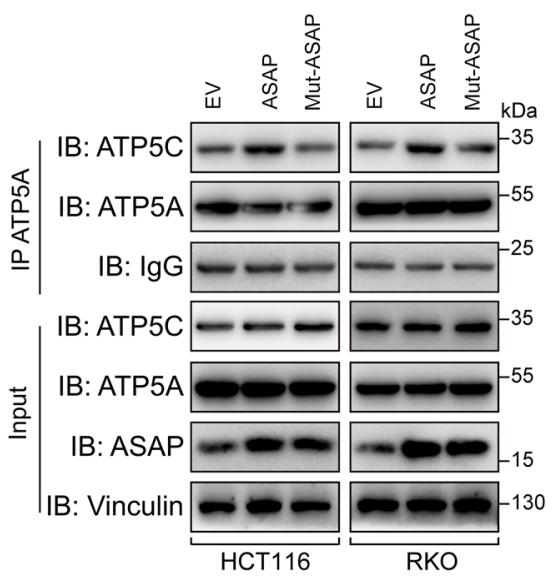

$\mathbf{F}$

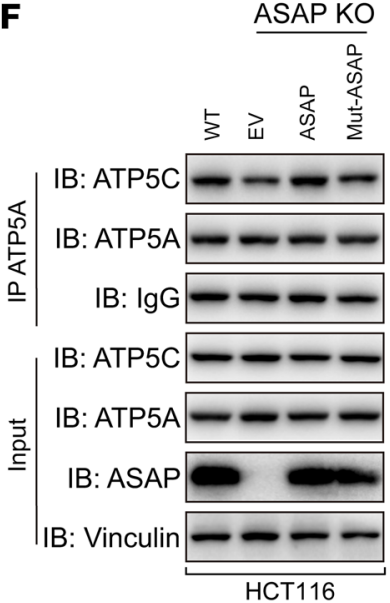

ASAP KO

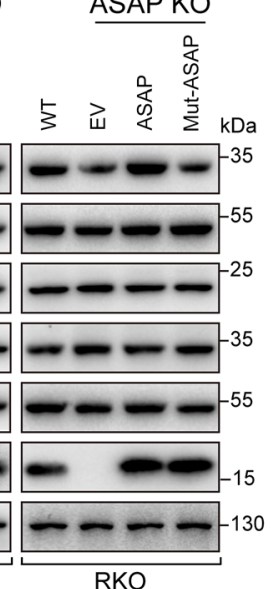

G

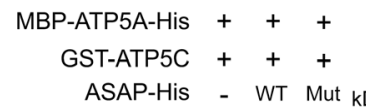
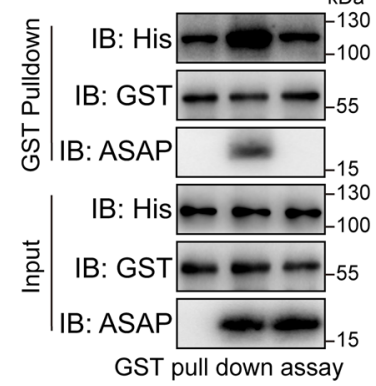

Figure 6. Identification of key residues that contribute to ASAP-ATP synthase interaction. (A) WT or mutant ASAP was cooverexpressed with SFBATP5A in HEK293T cells. Co-IP assays showed that K79A mutation on ASAP abolished the interaction between ASAP and ATP5A. (B) WT or mutant ASAP was cooverexpressed with SFB-ATP5C in HEK293T cells. Co-IP assays showed that D65A mutation on ASAP abolished the interaction between ASAP and ATP5C. (C) WT or mutant SFB-ATP5A was cooverexpressed with ASAP-His in HEK293T cells. Co-IP assays showed that D454A mutation on ATP5A abolished the interaction between ASAP and ATP5A. (D) WT or mutant SFB-ATP5C was cooverexpressed with ASAP-His in HEK293T cells. Co-IP assays showed that K197A mutation on ATP5C abolished the interaction between ASAP and ATP5C. (E) The endogenous interaction between ATP5A and ATP5C in HCT116 and RKO cells with ASAP overexpressed or Mut-ASAP was investigated using Co-IP assays. (F) The endogenous interaction between ATP5A and ATP5C in HCT116 and RKO cells with ASAP KO or ASAP restored was investigated using Co-IP assays. (G) In vitro pulldown assays were performed using bacterial purified MBP-ATP5A-His and CST-ATP5C in the presence of WT ASAP or mutant ASAP. Data are representative of 3 independent experiments (A-G).

cytoplasm of intestinal pit epithelial cells, scattered in the glands of the lamina propria, with mild to moderate intensity (Supplemental Figure 14C). The difference in expression pattern of ASAP between normal colonic epithelial tissues and CRC tissues suggested that ASAP might serve as a potential therapeutic target for CRC.

We then evaluated the association between ASAP and clinicopathological parameters, including patient survival outcomes. Upregulation of ASAP was observed in CRC patients with more lymph node metastasis (Supplemental Table 5). Kaplan-Meier analysis showed that patients with higher expression levels of ASAP in tumor had a poor prognosis (Figure 9M). In summary, our results suggested that ASAP was upregulated in CRC tissues and related to poor outcomes in patients.

\section{Discussion}

With the evolution of ribosome profiling, MS analysis, and in silico prediction, several databases of micropeptides translated from noncanonical ORFs have been established $(5-7,46)$. However, the specific functions and mechanisms of micropeptides have not been well elucidated. Recent studies $(11,13,14)$ have revealed the function of mitochondria-localized micropeptides in biosynthesis and bioenergy processes. In the current study, we characterized a micropeptide encoded by LINC00467 and identified its mitochondrial localization through mitochondria fraction assay and immunofluorescence staining. We found that ASAP regulated the production of mitochondrial ATP and enhanced the oxygen consumption of cancer cells. Mechanically, we observed that ASAP bound to the $\alpha$ and $\gamma$ subunits of ATP synthase and promoted their interaction, thereby enhancing the activity of ATP synthase. Given that ASAP colocalized to IMM, directly bound to ATP synthase, and presented little potential as a membrane-integrated protein, ASAP should be a mitoplast protein, but mainly interacting with the IMM-integrated ATP synthase. Taken together, our data show that mitochondrial-localized ASAP plays an important regulatory role in the mitochondrial protein machinery and may serve as a potential target for CRC treatment.

Previous studies reported that LINC00467 sponged microRNAs, thereby regulating the progression of leukemia (47), nonsmall cell lung cancer (48), esophageal carcinoma (49), and CRC (50). These studies indicated the oncogenic role of LINC00467, which is consistent with our findings. RNA transcripts could exert multifunctions through interacting with various biomolecules and encoding proteins. RNAs can also function in a context-dependent manner and present plastic physiological utility across somat- 

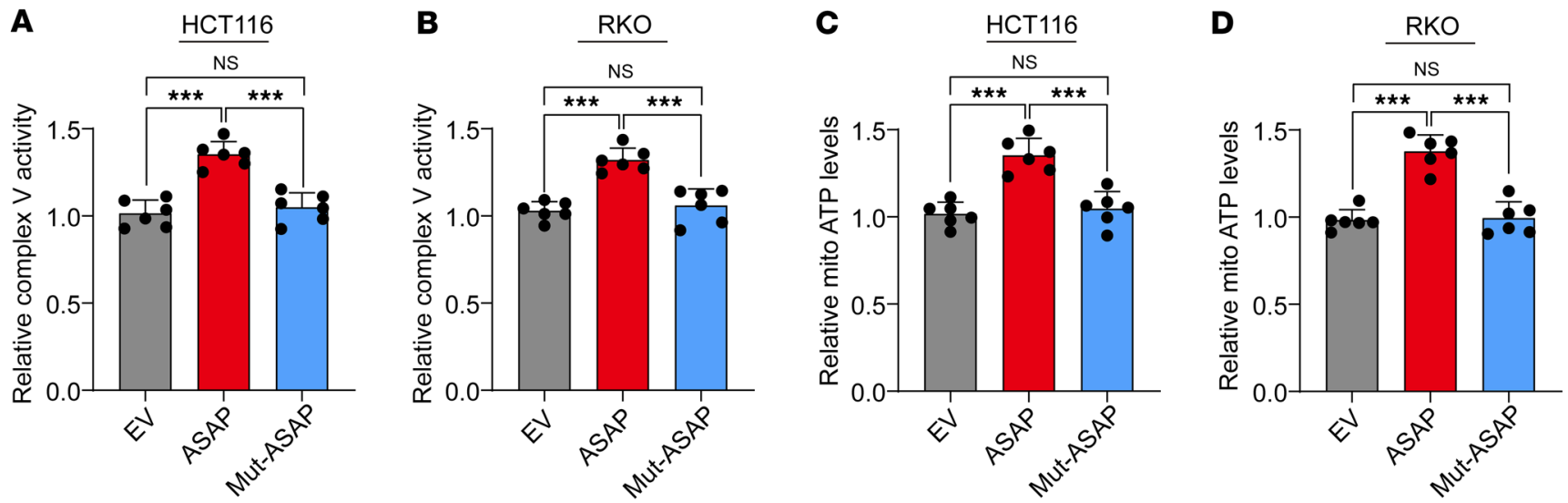
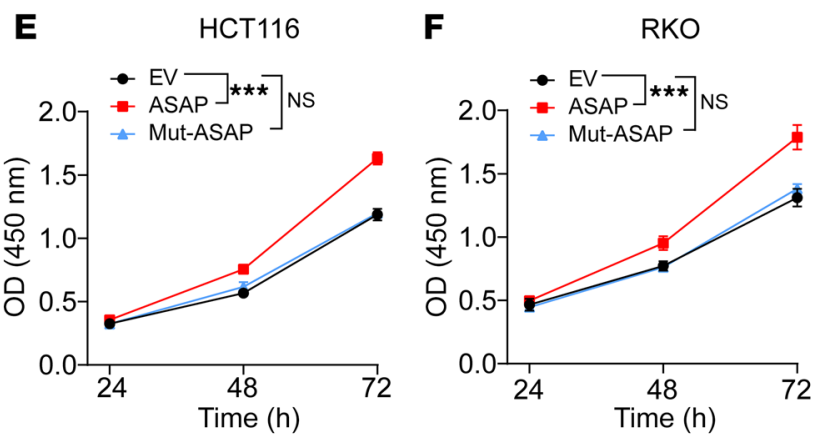

G HCT116
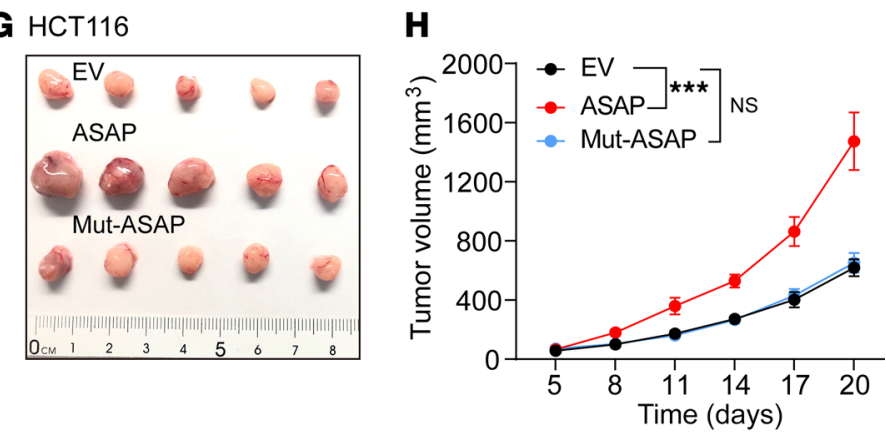

I

$\mathbf{J}$

HCT116
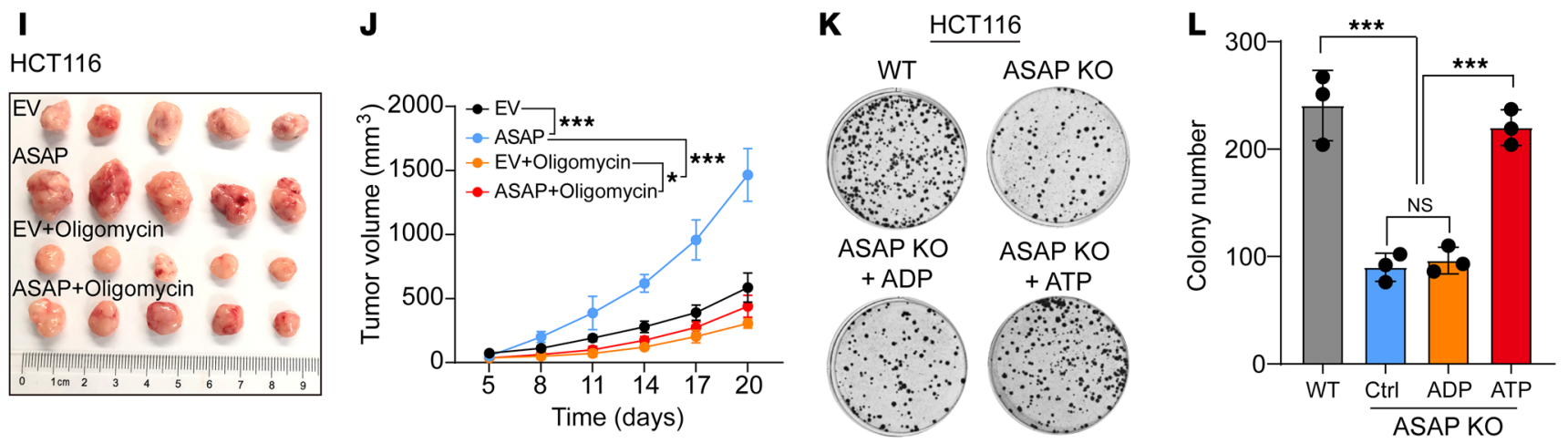

Figure 7. ASAP regulates CRC growth through mitochondrial ATP synthase. (A and B) The relative activities of respiratory complex V (ATP Synthase) were investigated in HCT116 (A) and RKO (B) cells with WT ASAP or Mut-ASAP overexpressed. Data are presented as mean \pm SD. $n=6$ biologically independent experiments. One-way ANOVA followed by Tukey's test. ${ }^{* *} P<0.001$. (C and $\left.\mathbf{D}\right)$ Relative mitochondrial ATP production was detected. HCT116 (C) and RKO (D) cells with ASAP overexpressed or Mut-ASAP overexpressed were treated with recording buffer (with $5 \mathrm{mM}$ 2-DG and 5 mM pyruvate) to determine ATP generation under mitochondrial ATP synthesis. Data are presented as mean \pm SD from $n=6$ biologically independent experiments. One-way ANOVA followed by Tukey's test. ${ }^{* *} P<0.001$. (E and $\mathbf{F}$ ) The CCK-8 assay was used to determine the proliferation rate of indicated cells. Data are presented as mean \pm SD. $n=3$ biologically independent experiments. Two-way ANOVA analysis. ${ }^{* *} P<0.001$. (G and $\left.\mathbf{H}\right)$ Xenograft mouse model using EV control, ASAP, or MutASAP-overexpressing HCT116 cells. In vivo generated tumors (C) and analyses of tumor growth and volume $(\mathbf{H})$ are shown. Two-way ANOVA analysis. ${ }^{* * *} P<$ 0.001. (I and J) Xenograft mouse model using EV control or ASAP-overexpressing HCT116 cells with or without the treatment of oligomycin. In vivo generated tumors (I) and analyses of tumor growth and volume (J) are shown. Data are presented as mean \pm SD from $n=5$ mice per group. Two-way ANOVA. ${ }^{*} P<0.05$; ${ }^{* * *} P<0.001$. (K and $\mathbf{L}$ ) Colony formation assay was performed on WT and ASAP KO HCT116 cells. KO cell lines were supplied with an additional 3 mM ADP or 3 mM ATP. Representative images (K) and statistical analysis (L) are shown. Each circle (K) shows a well of a 6-well plate with a diameter of around $3.4 \mathrm{~cm}$. Data are presented as mean \pm SD from $n=3$ biologically independent experiments. One-way ANOVA followed by Tukey's test. ${ }^{* * *} P<0.001$.

ic organs, such as the multifunction of GAS5 $(32,51,52)$. In this study, we found that LINC00467 encoded a micropeptide ASAP and LINC00467 with mutated ASAP start codon failed to regulate mitochondrial metabolism and cell proliferation, indicating that ASAP conferred the oncogenic effect to LINC00467 in CRC.

Metabolic reprogramming is a hallmark of cancer (15). During the past decades, the relative physiological impact of glycolysis versus OXPHOS is unclear. The upregulation of glycolysis does not necessarily determine the absolute anaerobic phenotype or downregulation of mitochondrial OXPHOS in cancer cells (53), and different tumors showed different levels of OXPHOS $(21,54$, 55). OXPHOS has been demonstrated to be upregulated in CRC $(19,20,56)$. A previous study demonstrated that OXPHOS covers about $80 \%$ ATP in RKO cells (57). Our findings provided a hint 
A

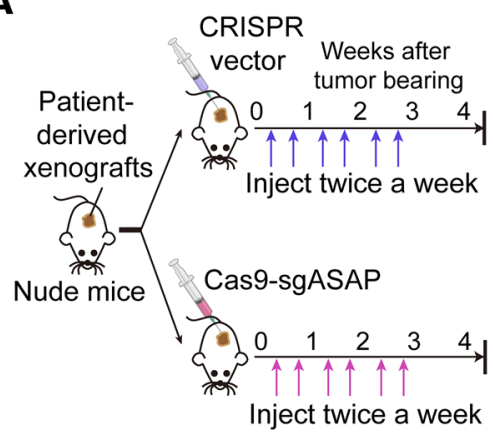

B

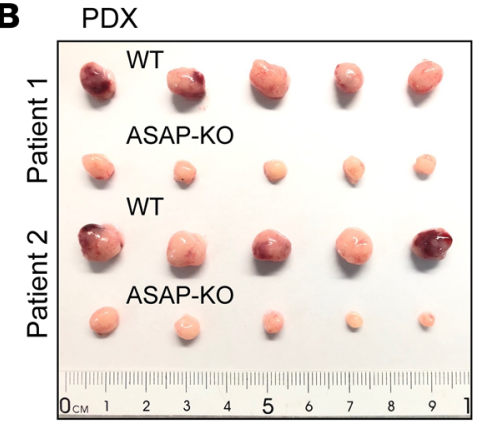

C
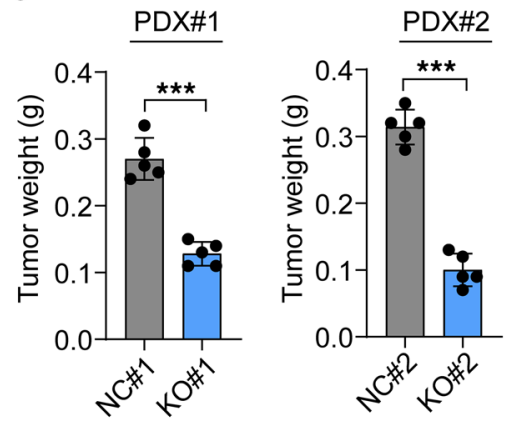

\section{E}
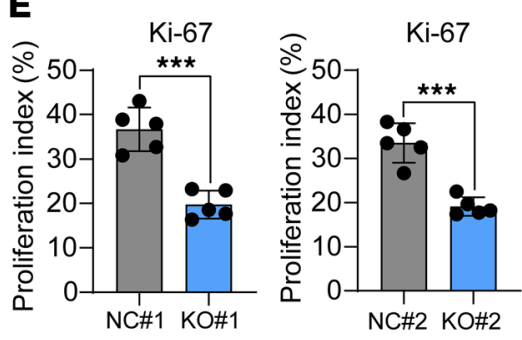
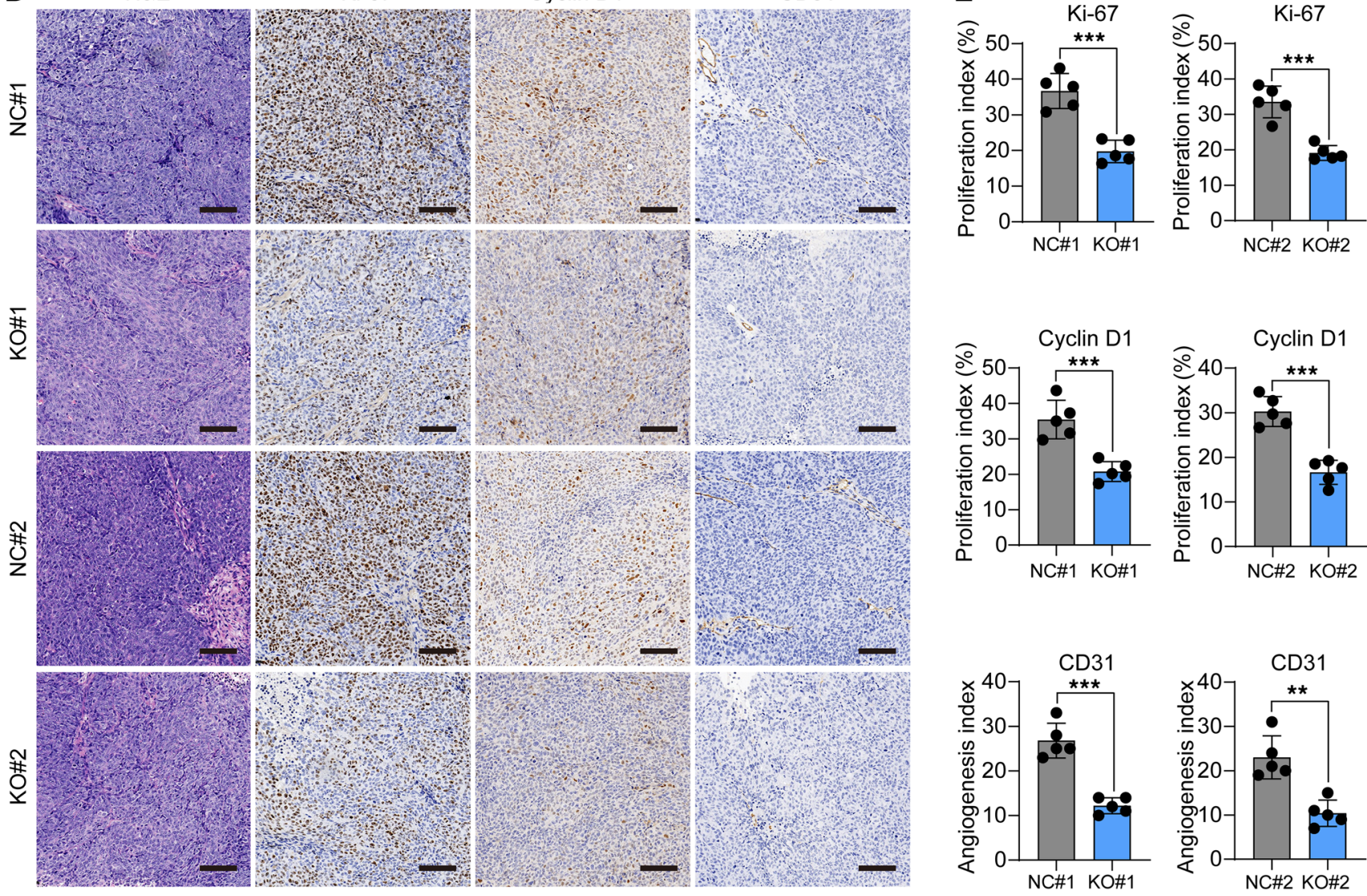

Figure 8. Targeting ASAP suppresses the growth of PDXs. (A) Schematic diagram showing the CRISPR/Cas9 system-based strategy to disrupt ASAP of PDXs. Three days after tumor inoculation, mice received 3 weeks of intratumoral injection of either ASAP-targeted CRISPR/Cas9 (Cas9-sgASAP) vector plus delivery buffer (purple arrows) or CRISPR v2 vector plus delivery buffer (blue arrows). (B and C) In vivo generated tumors (B) and tumor weight (C) of PDX models are shown. Independent samples, 2 -sided Student's $t$ test. ${ }^{* *} P<0.001$. (D) Representative IHC staining in tumors from PDX model are shown. Scale bars: $200 \mu \mathrm{m}$. (E) The relative intensities of IHC (Figure 8D) were quantified by Imagel software (version 1.51) in 10 random fields from 5 tumors in each group. Data are presented as mean \pm SD from $n=5$ tumor samples per group. Independent samples, 2-sided Student's $t$ test. ${ }^{* *} P<0.01$; ${ }^{* * *} P<0.001$.

that ASAP-promoted mitochondrial OXPHOS was critical for CRC progression. We observed a relatively high abundance of ASAP in CRC, which might be related to the cellular mitochondrial utility.

Given the ectopic activation of OXPHOS in CRC, targeting mitochondrial OXPHOS complexes may aid the clinical strategy of treating CRC $(58,59)$. Complex I is a therapeutic target for cancer treatment with promising effects (60-62). ATP synthase (complex V) is the crucial final step of OXPHOS, and several studies have reported some compounds targeting complex V. Gboxin accumulates inside mitochondria, targets complex V, rapidly blocks OXPHOS, and thereby exhibits promising antitumor activity in vivo (23). Leucinostatin A-derived LCS-7 can directly bind to the ATP synthase $\gamma$ subunit and decrease the growth of mouse xenograft tumors (24). As ASAP supports the function of ATP synthase, we designed a CRISPR/Cas9-based system to target ASAP and found that loss of ASAP markedly suppressed the growth of PDXs. Combining with the mild biological effect of ASAP knocking down in normal intestinal epithelial cells, our study indicates ASAP as a potential therapeutic target for OXPHOS-dependent CRC. 

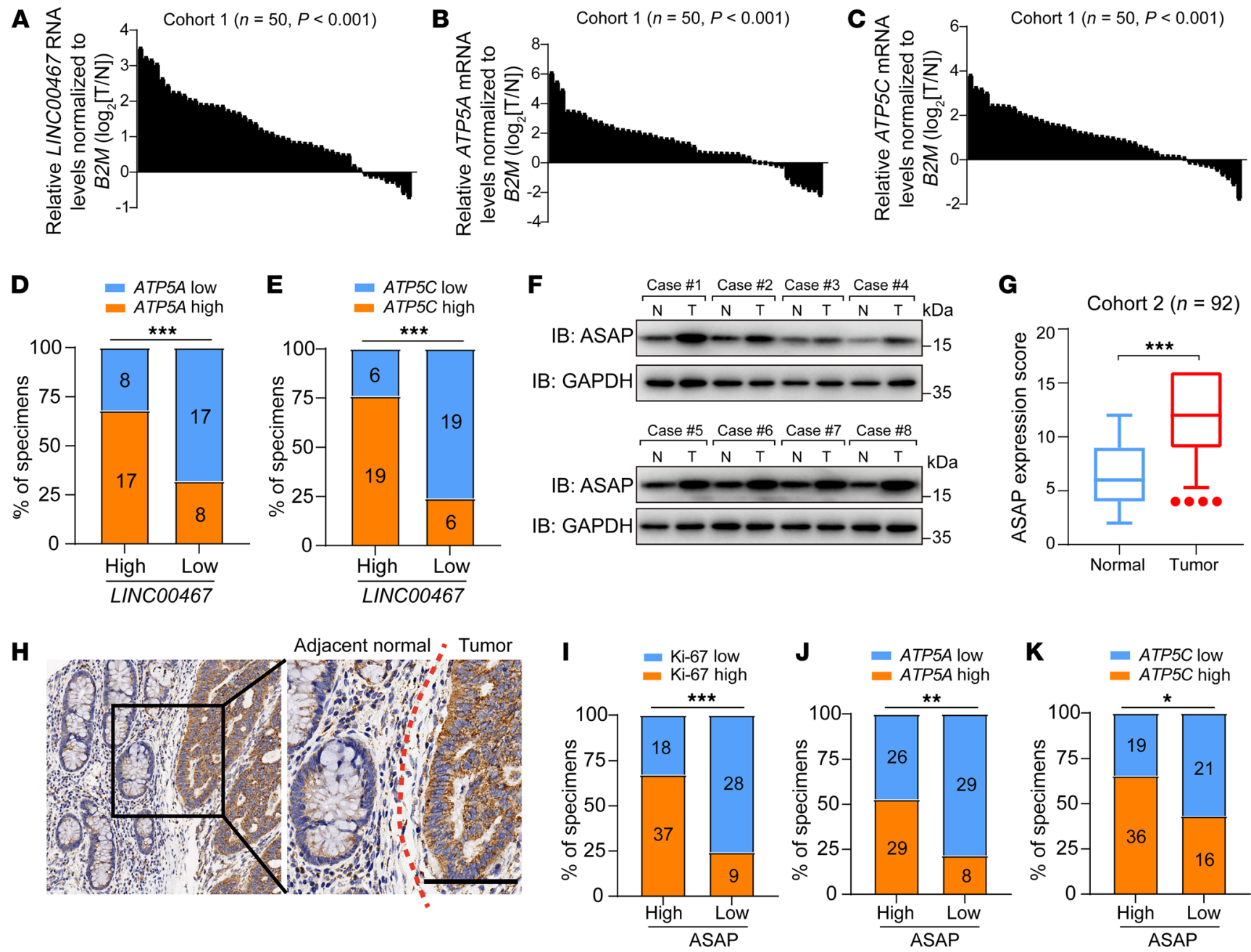

$\mathbf{L}$
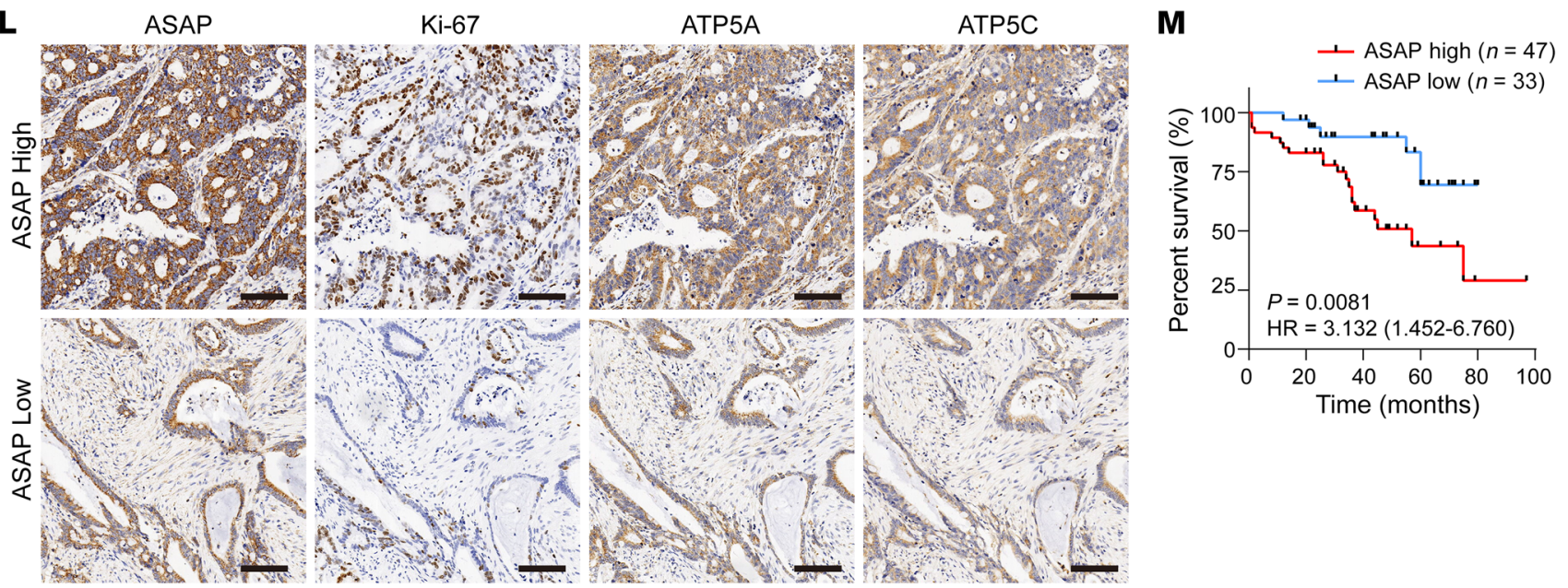
Figure 9. Expression of ASAP and LINC00467 predicts poor outcomes in CRC. (A-C) RNA levels of LINC00467 (A), ATP5A (B), and ATP5C (C) in CRC tissues and matched nontumor tissues in cohort $1(n=50)$ were detected by RT-qPCR in 3 independent experiments. Paired samples, 2-sided Student's $t$ test. ( $\mathbf{D}$ and $\mathbf{E}$ ) Percentages of specimens showing low or high LINC00467 expression related to levels of ATP5A (D) and ATP5C (E). Numbers in the bars indicate samples represented by each square. $\chi^{2}$ test. ${ }^{* * *} P<0.001$. (F) ASAP levels in randomly selected CRC and noncancerous tissues were detected by Western blot. Data are representative of 3 independent experiments. (G) Quantification of ASAP protein expression by scoring immunohistochemistry staining in CRC of cohort 2 . Bounds of boxes show the 25th and 75th percentiles, and the central lines in the boxes represent the median value. Whiskers show 5 th to 95 th percentiles. Outlying values, 4, 4, 4, 4 (tumor). Paired-samples 2-sided Student's $t$ test. ${ }^{* *} P<0.001$. (H) Representative images of ASAP staining on CRC and adjacent normal tissues. The red dotted line indicates the boundary between tumor and normal tissue. Scale bar: $200 \mu \mathrm{m}$. (I-K) Percentages of specimens showing low or high ASAP expression related to levels of Ki-67 (I), ATP5A (J), and ATP5C (K). Numbers in the bars indicate samples represented by each square. $\chi^{2}$ test. ${ }^{*} P<0.05$; ${ }^{* *} P<0.01$; ${ }^{* *} P<0.001$. (L) ASAP levels were significantly associated with expression of Ki-67, ATP5A, and ATP5C in primary human CRC specimens (cohort $2, n=92$ ). Two representative cases are shown. Scale bars: $200 \mu \mathrm{m}$. (M) CRC patients with follow-up data in cohort $2(n=80)$ were divided into 2 groups according to the expression of ASAP, and the overall survival curve was generated with Kaplan-Meier analysis and log-rank test.

ATP synthase in $H$. sapiens consists of Fo and F1 domains (39). The Fo domain consists of 8 subunits and a transmembrane ring, including ATP6 and ATP8 (63); the F1 domain consists of 5 subunits, $\alpha, \beta, \gamma, \delta$, and $\varepsilon$, encoded by ATP5A, ATP5B, ATP5C, ATP5D, and ATP5E, respectively (64). In this study, we tested the interplay among ASAP and all subunits of the F1 domain and found that ASAP directly bound to ATP synthase subunits $\alpha$ and $\gamma$ (ATP5A and ATP5C). Biochemical assays and molecular docking analysis demonstrated the interaction residues between ASAP and ATP5A or ATP5C. Future studies including nuclear magnetic resonance (NMR) or cocrystallization to show the interaction between ASAP and ATP synthase would be conducted to elucidate constitutive fundamental and molecular mechanism of ASAP-ATP synthase interactions, which might be helpful for screening ASAP-targeting compounds.

In summary, ASAP encoded by lncRNA LINC00467 plays an oncogenic role in CRC. ASAP is characterized as a mitochondria-localized micropeptide and functions as a regulator of ATP synthase to promote mitochondrial ATP production, OCR, and cell proliferation. Our findings provide insights for the study of mitochondrial-localized micropeptides and highlight the therapeutic potential of targeting ASAP in CRC.

\section{Methods}

Cell lines and cell culture. Human CRC cell lines (LoVo, HCT116, RKO, SW480) and a human embryonic kidney epithelial cell line (HEK293T) were purchased from ATCC. A normal human colon mucosal epithelial cell line (NCM460) was purchased from INCELL. HCT116 cells were cultured in McCoy's 5A medium (Genom), LoVo cells were cultured in F-12K medium (Genom), HEK293T, NCM460, and RKO cells were cultured in DMEM medium (Gibco, Thermo Fisher Scientific), and SW480 cells were cultured in RPMI 1640 medi- um (Gibco, Thermo Fisher Scientific) supplemented with 10\% fetal bovine serum (FBS) (Sijiqing). Cells were kept at $37^{\circ} \mathrm{C}$ in a $5 \%$ (vol/ vol) $\mathrm{CO}_{2}$-containing atmosphere. All cell lines were routinely tested and had negative results for mycoplasma contamination. No cell lines used in this study are found in the database of commonly misidentified cell lines (ICLAC and NCBI BioSample) based on short tandem repeat (STR) profiling performed by vendors.

Human samples. Two patient cohorts were recruited in the study. In cohort 1, 50 surgically resected CRC and matched adjacent noncancerous tissues were obtained from Sir Run Run Shaw Hospital. Tissues were used for quantitative reverse-transcriptase PCR (RT-qPCR) and Western blot analysis. In cohort 2, 92 patients with clinicopathologic characteristics who underwent surgery at the Second Affiliated Hospital of Zhejiang University School of Medicine and were histologically diagnosed with CRC were enrolled. Tissue microarrays were made using paraffin-embedded consecutive sections.

Reagents and antibodies. Chemical reagents DAPI (C0060), puromycin (P8230), PK (P9460), crystal violet (C8470), oligomycin (IO0460), 2-DG (D8930), and sodium pyruvate (SP0100) were purchased from Solarbio. MitoTracker Red (M7512) and Prestained Protein Ladder (26616) were purchased from Thermo Fisher. Antibodies against Tom20 (catalog 42406), cyclin D1 (catalog 55506), Ki67 (catalog 9449), CD31 (catalog 77699S), Vinculin (catalog 13901), SDHA (catalog 11998), $\alpha$-tubulin (catalog 2125S), and COX IV (catalog 11967S) were purchased from Cell Signaling Technology. Antibodies against His-tag (catalog M20001), Myc-tag (catalog M20002), GFP-tag (catalog M20004), GST-tag (catalog M20007), Flag-tag (catalog M20008), and GAPDH (catalog M20050) were purchased from Abmart. Antibodies against ATP5A (catalog 176569) and ATP5C (catalog 119686) were purchased from Abcam. The secondary antibodies used in Western blot, HRP goat anti-mouse IgG (H+L) (catalog BK-R050), and HRP goat anti-rabbit $\operatorname{IgG}(\mathrm{H}+\mathrm{L})$ (catalog BK-M050) were purchased from Bioker. Light-chain-specific mouse anti-rabbit IgG (catalog 58802S) was purchased from Cell Signaling Technology. The secondary antibodies used in immunofluorescence, goat anti-rabbit IgG H\&L (Alexa Fluor 488) (catalog 150077), goat anti-rabbit IgG H\&L (Alexa Fluor 594) (catalog 150080), goat anti-mouse IgG H\&L (Alexa Fluor 488) (catalog 150113), and goat anti-mouse IgG H\&L (Alexa Fluor 594) (catalog 150116) were purchased from Abcam. The polyclonal antibody against the ASAP micropeptide was produced to standard procedures by HuaBio. The polyclonal antibodies were obtained from inoculated rabbits and purified using affinity chromatography.

Polysome profiling. Polysome profiling was performed as we previously described (45) with minor modifications. $6 \times 10^{6}$ Cells were treated with $100 \mu \mathrm{g} / \mathrm{mL}$ cycloheximide (CHX) (Sigma-Aldrich) for 5 minutes. Cells were lysed with polysome lysis buffer $(15 \mathrm{mmol} / \mathrm{L}$ Tris$\mathrm{HCl}, 5 \mathrm{mmol} / \mathrm{L} \mathrm{MgCl}_{2}, 100 \mathrm{mmol} / \mathrm{L} \mathrm{KCl}, 2 \mathrm{mmol} / \mathrm{L}$ dithiothreitol, $1 \%$ Triton X-100, $100 \mu \mathrm{g} / \mathrm{mL} \mathrm{CHX).} \mathrm{Cell} \mathrm{lysates} \mathrm{were} \mathrm{centrifuged} \mathrm{at}$ $16,200 \mathrm{~g}$ at $4^{\circ} \mathrm{C}$ for 10 minutes. The supernatant was kept, and absorbance was measured at $260 \mathrm{~nm}$. Then the supernatant was loaded onto the top of a $5 \%$ to $50 \%$ sucrose gradient $(15 \mathrm{mmol} / \mathrm{L}$ Tris- $\mathrm{HCl}$, $5 \mathrm{mmol} / \mathrm{L} \mathrm{MgCl}_{2}, 100 \mathrm{mmol} / \mathrm{L} \mathrm{KCl}, 2 \mathrm{mmol} / \mathrm{L}$ dithiothreitol, $100 \mu \mathrm{g} /$ $\mathrm{mL} \mathrm{CHX)} \mathrm{and} \mathrm{centrifuged} \mathrm{at} \mathrm{210,000} \mathrm{g}$ at $4^{\circ} \mathrm{C}$ for 190 minutes (SW 41Ti ROTOR, Beckman). The gradient was collected into 12 fractions by monitoring RNA absorbance at $254 \mathrm{~nm}$. RNA in each fraction was extracted and quantified by RT-qPCR. The enrichment of lncRNAs on polysome was normalized to the input sample. 
Cloning procedures. The full lengths of LINC00467, ATP5A, ATP5B, ATP5C, ATP5D, ATP5E, SLC25A3, and HADHA were cloned by PCR. Each ORF was subcloned from the full-length of LINC00467. All eukaryotic expressed genes were cloned into an SFB lentiviral (S-tag, Flag-tag, and SBP-tag fused) vector and pcDNA3.1-Flag/Myc/ His vectors using ClonExpress II One Step Cloning Kit (Vazyme, C11201). All point mutations were generated by PCR overlapping. Bacterial expression vectors for MBP-His-tagged ATP5A and ATP5C were constructed by cloning into a pMBP28a vector. GST-tagged ASAP, ATP5A, and ATP5C were constructed by cloning into a pGEX-4T2 vector. Histagged ASAP was constructed by cloning into a pET28a vector.

Cell transfection and lentiviral-based gene transduction. All siRNA sequences were designed according to the siRNA Selection Program (http://sirna.wi.mit.edu/home.php/) (65) and commercially produced (GenePharma). Plasmids or siRNAs were performed using Lipofectamine 2000 (Thermo Fisher). Lentiviruses were produced in HEK293T cells with package vectors VSVG and psPAX2. The virus was collected to transduce target cells, followed by selection with puromycin $(1 \mu \mathrm{g} / \mathrm{mL})$. The sequence of siRNAs is listed in Supplemental Table 4.

CRISPR/Cas9-based genome editing. Designed gRNAs were inserted into lentiCRISPR v2 plasmid. Donor oligo was designed and commercially synthesized (Tsingke Biotech). The targeting vector was cotransfected with donor oligos into cells, followed by selection with puromycin $(1 \mu \mathrm{g} / \mathrm{mL})$. Single-cell clones were selected, and PCR was performed using genomic DNA as a template. The obtained PCR products were sequenced to confirm successful genome editing. The gRNA sequence used in the study is listed in the Supplemental Table 4.

Bioinformation analysis of ASAP. Homologous sequences of LINC00467 ORF1 were identified using NCBI-BLAST or BLAT search of the UCSC Genome Browser. Corresponding protein sequences were aligned using PRALINE (https://www.ibi.vu.nl/programs/ pralinewww/). Furtherevaluations of ASAPstructure and domainswere performed using the following online servers: C-I-TASSER (https:// zhanglab.ccmb.med.umich.edu/C-I-TASSER/), TMHMM (http:// www.cbs.dtu.dk/services/TMHMM/) and MITOPROT (https:// ihg.gsf.de/ihg/mitoprot.html).

GEO and TCGA database processing. Data from GSE22598 and GSE37364 were obtained from GEO. We calculated upregulated (fold change $>1.5$, adj $P<0.01$ ) differentially expressed genes (DEGs) with GEO2R from the GSE22598 gene expression-profiling data set, which contained the colorectal and adjacent normal samples $(n=17)$. We also retrieved the normalized expression matrix from GSE22598 and GSE37364 for the LINC00467 box plot. Ribosomal lncRNA was acquired from GSE143263 and annotated with GENCODE (version 30). Differentially expressed lncRNAs in TCGA COAD were acquired from the GEPIA web server (http://gepia.cancer-pku.cn/) using the differential expression analysis function with the threshold $\left(\left|\log _{2} \mathrm{FC}\right|\right.$ cutoff 0.01 and $q$ value cutoff 0.9 ). Genes containing an expression pattern similar to that of LINC00467 in colon cancer were identified by the similar genes detection function from GEPIA. GO Cellular Component (CC) analysis on obtained similar genes was carried out by $\mathrm{R}$ (https://www.r-project.org, version 3.5) and clusterProfiler packages.

RNA extraction and RT-qPCR. RNAs were extracted using TRIzol reagent (Takara). cDNA was synthesized using the iScript cDNA Synthesis Kit (Bio-Rad). The iTaq Universal SYBR Green Supermix qPCR Kit (Bio-Rad) was used to perform RT-qPCR. U6 and B2M were used as endogenous controls. Primers used are listed in Supplemental Table 4.
Western blot analysis. Cell lysate or tissue lysate was added to $12 \%$ SDS-PAGE and transferred to PVDF membranes (MilliporeSigma). ASAP micropeptide was more than $10 \mathrm{kDa}$, which could be separated by $12 \%$ SDS-PAGE. Membranes were incubated with primary antibodies overnight. HRP-linked goat anti-rabbit/mouse $\operatorname{IgG}$ was used as the secondary antibody. For ATP5A in the immunoprecipitation assay, light chain-specific secondary antibodies (Cell Signaling Technology, 58802S) were used. GAPDH, vinculin, and $\alpha$-tubulin were used as loading controls. The blots were visualized by ECL. Blot images were obtained using Image Lab software, version 4.1 (Bio-Rad).

Recombinant protein expression and purification. Recombinant proteins were expressed in Escherichia coli strain BL21-CodonPlus (DE3)RIPL (Agilent Technologies) and purified using Ni-NTA Sefinose Resin (Sangon Biotech) or GST-Sefinose Resin (Sangon Biotech). The concentration and purity of recombinant proteins were measured by SDS-PAGE and Coomassie staining (Supplemental Figure 15) with the standard BSA gradient.

Cell lysis and immunoprecipitation assay. Cells were collected and lysed. Supernatants were obtained by centrifugation at $13,000 \mathrm{~g}$ for 10 minutes at $4^{\circ} \mathrm{C}$. For immunoprecipitation assay, indicated antibodies and control IgG were added to the lysates. After incubation at $4^{\circ} \mathrm{C}$ for 2 hours with rotation, $10 \mu \mathrm{L}$ protein $\mathrm{A} / \mathrm{G}$ magnetic beads (Pierce, 88802) were added to the lysates, followed by incubation for another 2 hours. Then beads were washed with NETN buffer $(25 \mathrm{mM}$ Tris- $\mathrm{HCl}, 100 \mathrm{mM} \mathrm{NaCl}, 1 \mathrm{mM}$ EDTA, and $0.5 \mathrm{mM}$ dithiothreitol, $\mathrm{pH}$ 8.0) 3 times. For tagged-protein immunoprecipitation, the primary antibody and the protein $A / G$ beads were replaced with Flag-M2 magnetic beads (MilliporeSigma, M8823) or S-protein agarose beads (MilliporeSigma, 69704). The bead-enriched proteins were detected by Western blot analysis.

In vitro protein pull-down assay. GST-tagged protein was incubated with MBP-tagged protein in $500 \mu \mathrm{L}$ of binding buffer $(50 \mathrm{mM}$ Tris$\mathrm{HCl}, 10 \%$ glycerol, $100 \mathrm{mM} \mathrm{KCl}, 5 \mathrm{mM} \mathrm{MgCl}_{2}, 10 \mathrm{mM} \beta$-mercaptoethanol, and $0.1 \%$ NP-40, $\mathrm{pH}$ 8.0) supplemented with corresponding MBP resin (Sangon Biotech) or GST resin (Sangon Biotech) at $4^{\circ} \mathrm{C}$ for 3 hours with gentle rotation. Then the resin was washed with NETN buffer 3 times. The resin was boiled with $2 \times$ SDS loading buffer, and the protein enriched in the resin was detected by Western blot analysis.

Subcellular fractionation. Cells were incubated in Lysis buffer I (20 mM HEPES, $10 \mathrm{mM} \mathrm{KCl,} 2 \mathrm{mM} \mathrm{MgCl}, 0.5 \% \mathrm{NP}-40$, protease inhibitor, and RNase inhibitor, $\mathrm{pH}$ 7.4) on ice for 20 minutes. Lysates were centrifuged at a low speed of $1500 \mathrm{~g}$ for 5 minutes, and the supernatant (after low-speed spin) was transferred to a new tube. Then the new tube was centrifuged at a high speed of $15,000 g$ for 5 minutes. The resulting supernatant (after high-speed spin) was set aside as the cytoplasmic fraction. The pellet was suspended with Lysis Buffer I and centrifuged at $1500 \mathrm{~g}$ for 3 minutes. This was repeated for 3 times. The final pellet was lysed in $100 \mu \mathrm{L}$ Lysis buffer II (Lysis buffer I plus $0.5 \mathrm{M} \mathrm{NaCl}$ ) for 15 minutes (nuclear fraction). The nuclear lysates were centrifuged at $4^{\circ} \mathrm{C}$ for 10 minutes at $15,000 \mathrm{~g}$, and the supernatant was regarded as a nuclear fraction. RNA was extracted from the cytoplasmic and nuclear fractions and detected by RT-qPCR. GAPDH is an mRNA that was used as a cytosolic transcript marker; XIST is an lncRNA mainly located in the nucleus that was used as a nuclear transcript marker.

MS analysis. MS was performed by Novogen. In brief, the stained protein band was cut, destained, and dehydrated. $100 \mathrm{mM}$ TEAB, $1 \mu \mathrm{g}$ trypsin and $1 / 300 \mathrm{CaCl}_{2}$ were added into the gel. Proteins were 
digested at $37^{\circ} \mathrm{C}$. After centrifugation, the supernatant was collected and purified. The separated peptides were analyzed by Orbitrap Exploris 480 matched with FAIMS (Thermo Fisher), with ion source of Nanospray Flex (Thermo Scientific), spray voltage of $2.1 \mathrm{~V}$, and ion transport capillary temperature of $320^{\circ} \mathrm{C}$. Data-dependent acquisition mode was adopted by MS. For the identification of ASAP interactors, the resulting spectra were searched against the UniProt database. A maximum of 2 missed cleavage sites was allowed. The candidate ASAP interactors are listed in the Supplemental Table 3. IMM-associated candidates are highlighted.

Mitochondria purification and PK gradient assay. Mitochondria purification was performed as we previously reported (32). Cells were washed with KPBS buffer (136 mM KCl, $10 \mathrm{mM} \mathrm{KH}_{2} \mathrm{PO}_{4}, \mathrm{pH} 7.25$ ) and pelleted down by centrifugation at $4^{\circ} \mathrm{C}$ at $1000 \mathrm{~g}$. The cell pellet was resuspended with $500 \mu \mathrm{L}$ KPBS and subjected to $2 \mathrm{~mL}$ Dounce homogenization. After 40 to 50 strokes, cell extraction was centrifuged at $4^{\circ} \mathrm{C}$ at $1000 \mathrm{~g}$ for 10 minutes. The supernatant was centrifuged at $4^{\circ} \mathrm{C}$ at $1000 \mathrm{~g}$ for 10 minutes. The resulting supernatant was centrifuged at $4^{\circ} \mathrm{C}$ at $13,000 \mathrm{~g}$ for 10 minutes to obtain the LMF pellet (regarded as mitochondria and lysosome mixture), and the supernatant was set aside as the cytosol fraction. The LMF pellet was resuspended and incubated with $1 \mu \mathrm{L}$ TOM 20 antibody at $4^{\circ} \mathrm{C}$ for 2 hours with rotation, followed by the addition of $10 \mu \mathrm{L}$ protein $\mathrm{A} / \mathrm{G}$ magnetic beads for further incubation at $4^{\circ} \mathrm{C}$ for an additional 1 hour. The bead-enriched mitochondria were washed by pipetting $10 \times$ in 500 $\mu \mathrm{L}$ KPBS. The resulting bead complex was the purified mitochondrial fraction. For PK gradient assay, mitochondria were incubated with a range of $\mathrm{PK}$ concentrations from $1 \mathrm{ng} / \mathrm{mL}$ to $1 \mathrm{mg} / \mathrm{mL}$ at $37^{\circ} \mathrm{C}$ and then analyzed by Western blot. Mitochondria resuspended in RIPA were analyzed as full mitochondrial lysate control. PMSF ( $1 \mathrm{mM}$ final concentration) and proteinase inhibitors (Roche) were added to terminate PK digestion. The mitochondria could be solubilized by Triton $\mathrm{X}-100$ at a final concentration of $1 \%$.

Immunohistochemistry. The paraffin-embedded tissues were deparaffinized and rehydrated, followed by antigen retrieval in a cooker with $0.01 \mathrm{M}$ citrate buffer ( $\mathrm{pH}$ 6.0). Slides were incubated with primary antibodies overnight at $4^{\circ} \mathrm{C}$, and immunodetection was performed using HRP secondary antibody and DAB developer. The quantification of IHC staining density was measured using ImageJ software (version 1.51, NIH). For tissue microarrays, expression was evaluated by a pathologist and an investigator independently with an immunoreactivity score (IRS) (66), which was combined with a score of the percentage of positive cells (1, 0\% 25\%; 2, 25\% 50\%; 3, 50\% 75\%; and $4,75 \% \sim 100 \%$ ) and a score of the staining intensity (1, no staining of cells; 2 , mild staining; 3 , moderate staining; and 4, marked staining).

Immunofluorescence. GFP fluorescence in live cells was directly visualized. For other proteins, cells were fixed with $4 \%$ paraformaldehyde, permeabilized with $0.2 \%$ Triton X-100, blocked with 3\% BSA, and incubated with primary antibodies at $4^{\circ} \mathrm{C}$ overnight, and subsequently incubated with anti-rabbit (or mouse) IgG (Alexa Fluor 594 or 488 conjugated). Nuclei were counterstained with DAPI, and images were acquired with an FV3000 (Olympus) confocal microscope. For each channel, all images were acquired with the same settings. The high-solution image was obtained using a Leica TCS SP8 microscope with Lightning mode.

Colony formation assay. Indicated cells were seeded in a 6-well plate at a density of 300 cells per well. The cells were cultured for 10 to 14 days. Cells were fixed using $4 \%$ paraformaldehyde and stained with $0.5 \%$ crystal violet. The colony numbers were counted under a microscope $(n=3)$.

Cell proliferation assay. $5 \times 10^{3}$ Indicated cells were plated in 96-well plates and cultured in medium supplemented with 10\% FBS. The cell number was indicated by CCK- 8 assay (RiboBio) at the indicated time point. Experiments were performed in triplicate.

Measurement of cellular and mitochondrial ATP. The CellTiter-Glo Luminescent Cell Viability Assay Kit (Promega, G7570) was used for the measurement of cellular and mitochondrial ATP levels. Equal cells (cultured in different dishes) were seeded in a 96-well plate with 4 replicate wells for each cell line. After 18 hours of inoculation, 100 $\mu \mathrm{L}$ ATP reagent was added into each well and mixed. The plate was incubated for 10 minutes at room temperature, and the luminescence was measured on a luminometer. The values were normalized to the control group well in each individual experiment. For measurement of mitochondrial ATP levels, indicated cells were treated with recording buffer (with $5 \mathrm{mM}$ 2-DG and $5 \mathrm{mM}$ pyruvate) for 2 hours to determine ATP generation under mitochondrial ATP synthesis. Experiments were repeated 6 times.

Measurement of cell OCR. The OCR was determined using Seahorse Bioscience XF-24 Extracellular Flux Analyzer. $1 \times 10^{4}$ Cells were seeded in XF24-well cell culture microplates overnight. Different inhibitors (Agilent Technologies, 103015-100) were injected at the indicated time points: $1.0 \mu \mathrm{M}$ oligomycin was added to assess respiration related to ATP turnover; $0.5 \mu \mathrm{M}$ carbonyl cyanide 4-(trifluoromethoxy) phenylhydrazone (FCCP) was used to induce maximal respiration; rotenone/antimycin $\mathrm{A}$ (Rot/Anti-A) was added at a final concentration of $1.0 \mu \mathrm{M}$ to inhibit electron transport to detect the nonmitochondrial basal respiration level.

Measurement of ATP synthase activity. ATP synthase (complex V) activities were measured using the ATP Synthase Enzyme Activity Microplate Assay Kit (Abcam, ab109714) according to the manufacturer's instructions. Briefly, freshly isolated mitochondria were resuspended in PBS supplemented with 10\% detergent. Protein concentrations of these mitochondrial lysates were determined, and 25 $\mu \mathrm{g}$ mitochondrial protein was used per reaction. The ATP synthase enzyme was immunocaptured within the wells of the microplate, and the enzyme activity was measured by monitoring the decrease in absorbance at $340 \mathrm{~nm}$. Each cell line was measured with 4 replicate wells, and the values were normalized to the control group well in each individual experiment. Experiments were repeated 6 times.

Peptide structure prediction and molecular docking. The 3D structures of the ASAP peptide were generated using the C-I-TASSER web server. Molecular docking was performed as previously described (11). The structure of B. taurus ATP synthase, which is highly homologous with $H$. sapiens ATP synthase, was obtained from the PDB database (4ASU; ref. 41). The ClusPro server (40) was used for molecular docking of ASAP and ATP synthase. The docked structures were analyzed using the UCSF Chimera package.

Xenograft tumor model. Four-week-old female nude mice obtained from Shanghai SLRC Experimental Animal Center were maintained under specific pathogen-free conditions. Indicated cells were prepared at a density of $3.0 \times 10^{6}$ cells $/ 100 \mu \mathrm{L}$ and were subcutaneously injected into the right posterior flank of the mice. For oligomycin treatment, indicated cells were treated with $1 \mu \mathrm{g} / \mathrm{mL}$ oligomycin for 24 hours, washed, and harvested for subcutaneous inoculation. Tumor growth was 
observed every 3 days, and the volume was calculated by the following formula: volume $=\left(\right.$ length $\times$ width $\left.^{2}\right) / 2$. Mice were sacrificed when they met the institutional euthanasia criteria for tumor size and overall health condition. The tumors were removed and collected for further analysis.

PDX model. CRC surgical specimens were separated into small pieces $(2-3 \mathrm{~mm})$ and subcutaneously transplanted into 4-week-old female nude mice obtained from the Shanghai SLRC Experimental Animal Center. For the CRISPR/Cas9-based strategy to disrupt ASAP, mice were randomly assigned to groups 3 days after inoculation. Mice were treated with either ASAP-targeted CRISPR/Cas9 (Cas9-sgASAP, $2.5 \mu \mathrm{g}$ for each mouse) vector plus polyethylenimine (PEI) (SigmaAldrich) delivery buffer or CRISPR v2 vector plus delivery buffer as control via intratumoral injection twice per week. Tumor growth was observed every 5 days, and volume was calculated by the following formula: volume $=\left(\right.$ length $\times$ width $\left.^{2}\right) / 2$. Mice were sacrificed when they met the institutional euthanasia criteria for tumor size and overall health condition. The tumors were removed and collected for further analysis.

Statistics. Prism 8.0 software (GraphPad) was used to analyze data. The experiment was set up to use 3 to 5 samples/repeats for each experiment/group/condition. Representative images for IF staining, IHC staining, and immunoblot are shown. Results are reported as the mean value \pm SD of at least 3 independent experiments. Student's $t$ test was used to analyze the differences between 2 groups, 1-way ANOVA or 2-way ANOVA analysis was used to compare the differences from more than 2 groups, and $\chi^{2}$ test was used to compare the distribution of observations/frequencies in different categories. Overall survival curves were generated using the Kaplan-Meier method and estimated by the log-rank test. $P$ values of less than 0.05 were regarded as statistically significant.

Study approval. All patients providing specimens gave written, informed consent. Experiments were approved by the ethics committees of Sir Run Run Shaw Hospital and the Second Affiliated Hospital of Zhejiang University School of Medicine. Animal experimental protocols in the study were approved by the Committee of Animal Ethics of Zhejiang University.

\section{Author contributions}

LW, SC, and AL designed and supervised the study. QG, DJ, and DC performed most experiments with the assistance of CS, JL, JH, LJY, and LS. YQ performed most bioinformatics analysis. QG, DJ, DC, SC, AL, and LW analyzed and interpreted the data. QG drafted the manuscript. LW, SC, and AL critically revised the manuscript.

\section{Acknowledgments}

We thank Xintao Tu (University of Texas Southwestern Medical Center) for kindly editing the manuscript. We thank Baizhou Li (Zhejiang University) for the analysis of IHC results. We thank Qianqian Zhuang (Zhejiang University) for helpful discussion about mitochondria function assays. This project was financially supported by the National Natural Science Foundation of China (82072623 to LW; 81872300 to AL) and the Zhejiang Provincial Natural Science Foundation (Z22H168722 to LW; LGD21H160002 to SC).

Address correspondence to: Liangjing Wang, Department of Gastroenterology, the Second Affiliated Hospital of Zhejiang University School of Medicine, Hangzhou, 310009, China. Phone: 86.0571.89713905; Email: wangljzju@zju.edu.cn. Or to: Shujie Chen, Department of Gastroenterology, Sir Run Run Shaw Hospital, School of Medicine, Zhejiang University, Hangzhou, 310016, China. Phone: 86.0571.86006788; Email: chenshujie77@zju.edu. cn. Or to: Aifu Lin, College of Life Sciences, Zhejiang University, Hangzhou, 310058, China. Phone: 86.0571.88981750; Email: linaifu@zju.edu.cn.
1. Sung H, et al. Global Cancer Statistics 2020: GLOBOCAN estimates of incidence and mortality worldwide for 36 cancers in 185 countries. $C A$ Cancer JClin. 2021;71(3):209-249.

2. Brenner $\mathrm{H}$, et al. Prevention, early detection, and overdiagnosis of colorectal cancer within 10 years of screening colonoscopy in Germany. Clin Gastroenterol Hepatol. 2015;13(4):717-723.

3. Statello L, et al. Gene regulation by long noncoding RNAs and its biological functions. Nat Rev Mol Cell Biol. 2021;22(2):96-118.

4. Li W, et al. Enhancers as non-coding RNA transcription units: recent insights and future perspectives. Nat Rev Genet. 2016;17(4):207-223.

5. van Heesch S, et al. The translational landscape of the human heart. Cell. 2019;178(1):242-260.

6. Chen J, et al. Pervasive functional translation of noncanonical human open reading frames. Science. 2020;367(6482):1140-1146.

7. Prensner JR, et al. Noncanonical open reading frames encode functional proteins essential for cancer cell survival. Nat Biotechnol. 2021;39(6):697-704.

8. Anderson DM, et al. A micropeptide encoded by a putative long noncoding RNA regulates muscle performance. Cell. 2015;160(4):595-606.

9. Matsumoto A, et al. mTORC1 and muscle regeneration are regulated by the
LINC00961-encoded SPAR polypeptide. Nature. 2017;541(7636):228-232.

10. Zhu S, et al. An oncopeptide regulates m(6)A recognition by the $\mathrm{m}(6)$ A reader IGF2BP1 and tumorigenesis. Nat Commun. 2020;11(1):1685.

11. Huang $N$, et al. An upstream open reading frame in phosphatase and tensin homolog encodes a circuit breaker of lactate metabolism. Cell Metab. 2021;33(1):128-144.

12. Makarewich CA, et al. MOXI is a mitochondrial micropeptide that enhances fatty acid $\beta$-oxidation. Cell Rep. 2018;23(13):3701-3709.

13. Chugunova A, et al. LINCO0116 codes for a mitochondrial peptide linking respiration and lipid metabolism. Proc Natl Acad Sci U S A. 2019;116(11):4940-4945.

14. Stein CS, et al. Mitoregulin: a lncRNA-encoded microprotein that supports mitochondrial supercomplexes and respiratory efficiency. Cell Rep. 2018;23(13):3710-3720.

15. Hanahan D, Weinberg RA. Hallmarks of cancer: the next generation. Cell. 2011;144(5):646-674.

16. Viale A, et al. Oncogene ablation-resistant pancreatic cancer cells depend on mitochondrial function. Nature. 2014;514(7524):628-632.

17. Sriskanthadevan S, et al. AML cells have low spare reserve capacity in their respiratory chain that renders them susceptible to oxidative meta- bolic stress. Blood. 2015;125(13):2120-2130.

18. Chekulayev V, et al. Metabolic remodeling in human colorectal cancer and surrounding tissues: alterations in regulation of mitochondrial respiration and metabolic fluxes. Biochem Biophys Rep. 2015;4:111-125.

19. Kaldma A, et al. An in situ study of bioenergetic properties of human colorectal cancer: the regulation of mitochondrial respiration and distribution of flux control among the components of ATP synthasome. Int J Biochem Cell Biol. 2014;55:171-186.

20. Sun $X$, et al. Increased mtDNA copy number promotes cancer progression by enhancing mitochondrial oxidative phosphorylation in microsatellite-stable colorectal cancer. Signal Transduct Target Ther. 2018;3:8.

21. Ashton TM, et al. Oxidative phosphorylation as an emerging target in cancer therapy. Clin Cancer Res. 2018;24(11):2482-2490.

22. Junge W, Nelson N. ATP synthase. Annu Rev Biochem. 2015;84:631-657.

23. Shi Y, et al. Gboxin is an oxidative phosphorylation inhibitor that targets glioblastoma. Nature. 2019;567(7748):341-346.

24. Ohishi T, et al. Inhibition of mitochondria ATP synthase suppresses prostate cancer growth through reduced insulin-like growth factor-1 
secretion by prostate stromal cells. Int J Cancer. 2020;146(12):3474-3484.

25. Rombel IT, et al. ORF-FINDER: a vector for high-throughput gene identification. Gene. 2002;282(1-2):33-41.

26. Slavoff SA, et al. Peptidomic discovery of short open reading frame-encoded peptides in human cells. Nat Chem Biol. 2013;9(1):59-64.

27. D'Lima NG, et al. A human microprotein that interacts with the mRNA decapping complex. Nat Chem Biol. 2017;13(2):174-180.

28. He X, et al. Knock-in of large reporter genes in human cells via CRISPR/Cas9-induced homology-dependent and independent DNA repair. Nucleic Acids Res. 2016;44(9):e85.

29. Parras-Molto M, et al. Classification of protein motifs based on subcellular localization uncovers evolutionary relationships at both sequence and functional levels. BMC Bioinformatics. 2013;14:229.

30. Bouayad D, et al. Nuclear-to-cytoplasmic relocalization of the proliferating cell nuclear antigen (PCNA) during differentiation involves a chromosome region maintenance 1 (CRM1)dependent export and is a prerequisite for PCNA antiapoptotic activity in mature neutrophils. J Biol Chem. 2012;287(40):33812-33825.

31. Schmidt O, et al. Mitochondrial protein import: from proteomics to functional mechanisms. Nat Rev Mol Cell Biol. 2010;11(9):655-667.

32. Sang L, et al. Mitochondrial long non-coding RNA GAS5 tunes TCA metabolism in response to nutrient stress. Nat Metab. 2021;3(1):90-106.

33. Yu J, et al. PRICKLE3 linked to ATPase biogenesis manifested Leber's hereditary optic neuropathy. JClin Invest. 2020;130(9):4935-4946.

34. Black M, et al. FOXM1 nuclear transcription factor translocates into mitochondria and inhibits oxidative phosphorylation. Mol Biol Cell. 2020;31(13):1411-1424.

35. Spinelli JB, Haigis MC. The multifaceted contributions of mitochondria to cellular metabolism. Nat Cell Biol. 2018;20(7):745-754.

36. Moyer MP, et al. NCM460, a normal human colon mucosal epithelial cell line. In Vitro Cell Dev Biol Anim. 1996;32(6):315-317.

37. Boulet A, et al. The mammalian phosphate carrier SLC25A3 is a mitochondrial copper transporter required for cytochrome $c$ oxidase biogenesis. J Biol Chem. 2018;293(6):1887-1896.

38. Miklas JW, et al. TFPa/HADHA is required for fatty acid beta-oxidation and cardiolipin re- modeling in human cardiomyocytes. Nat Commun. 2019;10(1):4671.

39. Pinke G, et al. Cryo-EM structure of the entire mammalian F-type ATP synthase. Nat Struct Mol Biol. 2020;27(11):1077-1085.

40. Kozakov D, et al. The ClusPro web server for protein-protein docking. Nat Protoc. 2017;12(2):255-278.

41. Rees DM, et al. Structural evidence of a new catalytic intermediate in the pathway of ATP hydrolysis by F1-ATPase from bovine heart mitochondria. Proc Natl Acad Sci U S A. 2012;109(28):11139-11143.

42. Qian Y, et al. Extracellular ATP is internalized by macropinocytosis and induces intracellular ATP increase and drug resistance in cancer cells. Cancer Lett. 2014;351(2):242-251.

43. Chaudry IH. Does ATP cross the cell plasma membrane. Yale J Biol Med. 1982;55(1):1-10.

44. Yi L, Li J. CRISPR-Cas9 therapeutics in cancer: promising strategies and present challenges. Biochim Biophys Acta. 2016;1866(2):197-207.

45. Zhuo W, et al. Long noncoding RNA GMAN, up-regulated in gastric cancer tissues, is associated with metastasis in patients and promotes translation of ephrin A1 by competitively binding GMAN-AS. Gastroenterology. 2019;156(3):676-691.

46. Lu S, et al. A hidden human proteome encoded by 'non-coding' genes. Nucleic Acids Res. 2019;47(15):8111-8125.

47. Lu J, et al. Long noncoding RNA LINC00467 facilitates the progression of acute myeloid leukemia by targeting the miR-339/SKI pathway. Leuk Lymphoma. 2021;62(2):428-437.

48. Zhu Y, et al. LINC00467 is up-regulated by TDG-mediated acetylation in non-small cell lung cancer and promotes tumor progression. Oncogene. 2020;39(38):6071-6084.

49. Liu Z, et al. LncRNA LINC00467 acted as an oncogene in esophageal squamous cell carcinoma by accelerating cell proliferation and preventing cell apoptosis via the miR-485-5p/DPAGT1 axis. J Gastroenterol Hepatol. 2021;36(3):721-730.

50. He X, et al. Up-regulation of LINCO0467 promotes the tumourigenesis in colorectal cancer. JCancer. 2019;10(25):6405-6413.

51. Zhang XC, et al. YY1/LncRNA GAS5 complex aggravates cerebral ischemia/reperfusion injury through enhancing neuronal glycolysis. Neuropharmacology. 2019;158:107682.

52. Xu C, et al. Long non-coding RNA GAS5 controls human embryonic stem cell self-renewal by maintaining NODAL signalling. Nat Commun. 2016;7:13287.

53. Wallace DC. Mitochondria and cancer. Nat Rev Cancer. 2012;12(10):685-698.

54. Zu XL, Guppy M. Cancer metabolism: facts, fantasy, and fiction. Biochem Biophys Res Commun. 2004;313(3):459-465.

55. Reznik E, et al. Mitochondrial DNA copy number variation across human cancers. Elife. 2016;5:e10769.

56. Larman TC, et al. Spectrum of somatic mitochondrial mutations in five cancers. Proc Natl Acad Sci U S A. 2012;109(35):14087-14091.

57. Huang Q, et al. LYRM2 directly regulates complex I activity to support tumor growth in colorectal cancer by oxidative phosphorylation. Cancer Lett. 2019;455:36-47.

58. Zannella VE, et al. Reprogramming metabolism with metformin improves tumor oxygenation and radiotherapy response. Clin Cancer Res. 2013;19(24):6741-6750.

59. Kelly CJ, et al. Regulation of $\mathrm{O} 2$ consumption by the PI3K and mTOR pathways contributes to tumor hypoxia. Radiother Oncol. 2014;111(1):72-80.

60. Naguib A, et al. Mitochondrial complex I inhibitors expose a vulnerability for selective killing of pten-null cells. Cell Rep. 2018;23(1):58-67.

61. Liu X, et al. Metformin targets central carbon metabolism and reveals mitochondrial requirements in human cancers. Cell Metab. 2016;24(5):728-739.

62. Molina JR, et al. An inhibitor of oxidative phosphorylation exploits cancer vulnerability. Nat Med. 2018;24(7):1036-1046.

63. Guo H, et al. Atomic model for the dimeric $\mathrm{F}_{\mathrm{O}}$ region of mitochondrial ATP synthase. Science. 2017;358(6365):936-940.

64. He J, et al. Assembly of the membrane domain of ATP synthase in human mitochondria. Proc Natl Acad Sci U S A. 2018;115(12):2988-2993.

65. Bingbing Y, et al. siRNA Selection Server: an automated siRNA oligonucleotide prediction server. Nucleic Acids Res. 2004;32:W130-W134.

66. $\mathrm{Ge} \mathrm{Q}$, et al. Zic1 suppresses gastric cancer metastasis by regulating Wnt/ $\beta$-catenin signaling and epithelial-mesenchymal transition. FASEB J. 2020;34(2):2161-2172.

67. Yoshida M, et al. ATP synthase-a marvellous rotary engine of the cell. Nat Rev Mol Cell Biol. 2001;2(9):669-677. 